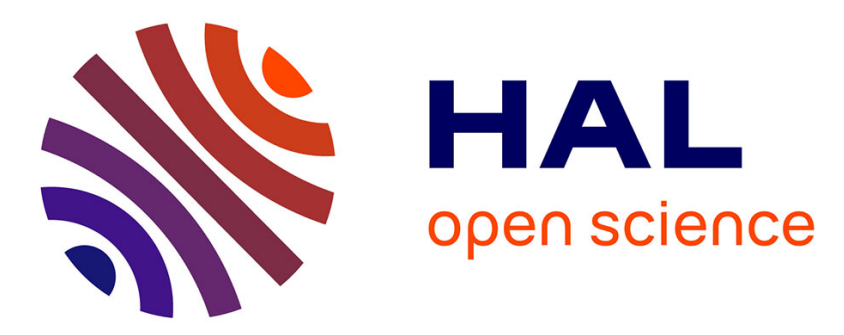

\title{
Asymptotic Arbitrage in Large Financial Markets With Friction
}

\author{
Emmanuel Lépinette, Lavinia Ostafe
}

\section{To cite this version:}

Emmanuel Lépinette, Lavinia Ostafe. Asymptotic Arbitrage in Large Financial Markets With Friction. 2011. hal-00591136v4

\section{HAL Id: hal-00591136 https://hal.science/hal-00591136v4}

Preprint submitted on 13 Apr 2012

HAL is a multi-disciplinary open access archive for the deposit and dissemination of scientific research documents, whether they are published or not. The documents may come from teaching and research institutions in France or abroad, or from public or private research centers.
L'archive ouverte pluridisciplinaire HAL, est destinée au dépôt et à la diffusion de documents scientifiques de niveau recherche, publiés ou non, émanant des établissements d'enseignement et de recherche français ou étrangers, des laboratoires publics ou privés. 


\title{
ASYMPTOTIC ARBITRAGE IN LARGE FINANCIAL MARKETS WITH FRICTION
}

\author{
By Emmanuel LEPINETTE*, ${ }^{*}$, LAVinia OSTAFE* \\ Paris-Dauphine University and University of Vienna
}

In the modern version of Arbitrage Pricing Theory suggested by Kabanov and Kramkov the fundamental financially meaningful concept is an asymptotic arbitrage. The "real world" large market is represented by a sequence of "models" and, though each of them is arbitrage free, investors may obtain non-risky profits in the limit. Mathematically, absence of the asymptotic arbitrage is expressed as contiguity of envelopes of the sets of equivalent martingale measures and objective probabilities. The classical theory deals with frictionless markets. In the present paper we extend it to markets with transaction costs. Assuming that each model admits consistent price systems, we relate them with families of probability measures and consider their upper and lower envelopes. The main result concerns the necessary and sufficient conditions for absence of asymptotic arbitrage opportunities of the first and second kinds expressed in terms of contiguity. We provide also more specific conditions involving Hellinger processes and give applications to particular models of large financial markets.

Acknowledgements The authors are grateful to the anonymous referee for the valuable comments and suggestions which improved the presentation.

1. Introduction. The idea to describe a financial market by a sequence of market models with a finite number of securities can be traced back to the paper [7] by Huberman who formalized intuitive arguments of Arbitrage Pricing Theory initiated by Ross, [18]. The famous conclusion of this theory is: under the absence of arbitrage, appropriately defined, the expected returns on assets are approximately linearly related to the factor loadings, "betas", proportional to the return covariances with the factors. In economic literature, the APT is considered as a substitute for the Capital Asset Pricing Model (CAPM) by Lintner and Sharp. The Ross-Huberman theory is

${ }^{*}$ The authors warmly express their thanks to Yuri Kabanov for his helpful discussions and advices.

${ }^{\dagger}$ The first author acknowledges the hospitality of Lavinia Ostafe and Walter Schachermayer at the University of Vienna where this work was completed.

AMS 2000 subject classifications: 60G44, G11-G13

Keywords and phrases: Large financial market, asymptotic arbitrage, transaction costs, contiguity, hedging theorem. 
single-period and uses a definition of arbitrage different from the one that is now standard. Its generalization to the standard continuous-time framework of modern mathematical finance was considered for a long time as a challenging problem of large importance.

This problem was solved in 1994 by Kabanov and Kramkov, [11], who suggested a concept of large financial market described by a sequence of "standard" financial market models with finite number of securities whose price processes admit martingale measures. They introduced new notions of Asymptotic Arbitrage of the First and Second Kind and, assuming that martingale measures are unique for each model, they established necessary and sufficient conditions for the absence of asymptotic arbitrage in terms of contiguity of the sequences of objective probabilities and martingale measures. As a particular example of application of their general approach, Kabanov and Kramkov considered a large Black-Scholes market where the stock prices are given by correlated geometric Brownian motions. For this case their general criteria give a result of the same type as the Ross-Huberman condition but involving instantaneous returns and covariances.

Significant progress in the theory was achieved in the paper by Klein and Schachermayer, [8], where the geometric functional analysis was used to obtain criteria of absence of asymptotic arbitrage for the case of incomplete market models when the martingale measures are not unique. The next step in the development of the general theory as well as in the understanding of financial framework was again done by Kabanov and Kramkov, [12]. They added several new criteria of absence of asymptotic arbitrage in terms of contiguity of sequences of upper and lower envelopes of martingale measures and objective probabilities. The technique of the proofs was based on the optional decomposition theorem. The criteria of Klein and Schachermayer was also obtained by an elegant use of the minimax theorem. Kabanov and Kramkov related their criteria with an extension of the Liptser-Shiryaev theory of contiguity of sequences of probability measures on filtered spaces in terms of the Hellinger processes. One should emphasize that Kabanov-Kramkov framework is very general and flexible. It covers discrete and continuous-time models, models with time horizons tending to infinity, etc. For the further development of the theory of large financial markets we send the reader to the articles [5], [15] but also [9] and [10].

In the present paper we extend the framework of large financial markets to the case of a market with friction. It is well known, in the theory of markets with proportional transaction costs the concept of martingale measures is not natural and is replaced by the notion of consistent price systems, i.e. the martingales evolving in the duals to the solvency cones expressed in physical 
units, [13]. The consistent price systems are vector objects. Nevertheless, the criteria of absence of asymptotic arbitrage can be formulated in terms of contiguity of objective probabilities and envelopes of measures naturally arising from consistent price systems. These are our principal results. We follow the lines of [12] but do not use the optional decomposition theorem (it has no analogue for models with transaction costs) but the hedging theorem. We use the abstract setting of the recent paper by Denis and Kabanov [3], which allows us to avoid detailed discussions on the structure of continuoustime models and cover both major approaches to the definition of the value processes, those of Kabanov and of Campi-Schachermayer [2].

Some examples are given. We extend the results of [12] to models with transaction costs: the one-stage APM by Ross, the large Black-Scholes market, and a two-asset model with infinite horizon.

\section{The Model: Definitions and Assumptions.}

2.1. General Model. The framework setting we present in this section is assumed to be satisfied by a sequence of markets of horizon dates $T$ (for the sake of simplicity, we omit the index $n$ ). We consider the general model of the paper [3] including the Kabanov and Campi-Schachermayer models with transaction costs. This is a continuous-time generalization of the discretetime financial model with transaction costs defined in Chapter 3, [13].

Let $\left(\Omega, \mathcal{F}, \mathbf{F}=\left(\mathcal{F}_{t}\right)_{t \leq T}, P\right)$ be a continuous-time stochastic basis verifying the usual conditions. We are given a pair of set-valued adapted processes $G=\left(G_{t}\right)_{t \leq T}$ and its positive dual $G^{*}=\left(G_{t}^{*}\right)_{t \leq T}$ whose values are closed cones in $\mathbf{R}^{\bar{d}}$, i.e.

$$
G_{t}^{*}(\omega)=\left\{y: y x \geq 0 \forall x \in G_{t}(\omega)\right\} .
$$

"Adapted" means that the graphs

$$
\left\{(\omega, x) \in \Omega \times \mathbf{R}^{d}: x \in G_{t}(\omega)\right\}
$$

are $\mathcal{F}_{t} \times \mathcal{B}\left(\mathbf{R}^{d}\right)$-measurable.

We assume that all the cones $G_{t}$ are proper, i.e. $G_{t} \cap\left(-G_{t}\right)=\{0\}$ or, equivalently, $\operatorname{int} G_{t}^{*} \neq \emptyset$. In a financial context it means that the efficient friction condition (EF) is fulfilled. We assume also that $G_{t}$ dominates $\mathbf{R}_{+}^{d}$, i.e. $G^{*} \backslash\{0\} \subset \operatorname{int} \mathbf{R}_{+}^{d}$. In a more specific financial setting (see [13]), the cones $G_{t}$ are the solvency cones when the portfolio positions are expressed in physical units.

imsart-aap ver. 2011/05/20 file: MafeLepinetteOstafe_02.04.tex date: April 3, 2012 
We are given a convex cone $\mathcal{Y}_{0}^{T}$ of optional $\mathbf{R}^{d}$-valued processes $\left(Y_{t}\right)_{t \leq T}$ with $Y_{0}=0$. We may interpret these processes as portfolios expressed in physical units.

Notations. We denote by $L^{0}\left(G_{t}, \mathcal{F}_{t}\right)$ the set of all $G_{t}$-valued $\mathcal{F}_{t}$-measurable random variables. A cone $G$ induces a natural order among $\mathbf{R}^{d}$-valued random variables. More precisely, for two $d$-dimensional random variables $Y$ and $Y^{\prime}$, we write $Y \geq_{G} Y^{\prime}$ if $Y-Y^{\prime} \in G$. The notation 1 stands for the vector $(1, \ldots, 1) \in \mathbf{R}_{+}^{d}$. Denote by $\mathcal{Y}_{0, b}^{T}$ the subset of $\mathcal{Y}_{0}^{T}$ formed by the processes $Y$ dominated from below in the sense of the partial orders generated by $\left(G_{t}\right)_{t \leq T}$, i.e. there is a constant $\kappa$ such that the process $Y+\kappa \mathbf{1}$ evolves in $G$. We also write $\mathcal{Y}_{0, b}^{T}(T)$ for the set of random variables $Y_{T}$ where $Y$ belongs to $\mathcal{Y}_{0, b}^{T}$. We denote by $\mathcal{M}_{0}^{T}\left(G^{*}\right)$ the set of all $d$-dimensional martingales $Z=\left(Z_{t}\right)_{t \leq T}$ with trajectories evolving in $G^{*}$, i.e. such that $Z_{t} \in G_{t}^{*}$ a.s. In the literature, such martingales are commonly called consistent price systems and strictly consistent price systems if they evolve in the interior of $G^{*}$.

Assumptions. Throughout the note we assume the following standing hypotheses on the sets $\mathcal{Y}_{0, b}^{T}(T)$ :

$$
\begin{aligned}
& \text { S1 : } E \xi Z_{T} \leq 0, \forall \xi \in \mathcal{Y}_{0, b}^{T}(T), Z \in \mathcal{M}_{0}^{T}\left(G^{*}\right) . \\
& \text { S2 : } \bigcup_{t \leq T} L^{\infty}\left(-G_{t}, \mathcal{F}_{t}\right) \subseteq \mathcal{Y}_{0, b}^{T}(T) .
\end{aligned}
$$

The hypotheses $\mathbf{S}_{1}$ and $\mathbf{S}_{2}$ adopted in this note allow us to avoid the unnecessary repetitions and do not provide the full description of continuoustime models with transaction costs. It is important to know only that these conditions are fulfilled for the known models, see [14], [2], [4].

Recall that in these financial models $\mathbf{S}_{1}$ holds because, if one calculates the current portfolio value using a price system $Z$ (that is a process from $\mathcal{M}_{0}^{T}\left(G^{*}\right)$ ), the resulting scalar process is a supermartingale. In a discretetime model, a portfolio process $\left(V_{t}\right)_{t \leq T}$ is such that $V_{t} \in \sum_{u=0}^{t} L^{0}\left(-G_{u}, \mathcal{F}_{u}\right)$ for all $t \leq T$. If $V \in \mathcal{Y}_{0, b}^{T}$ then $E\left(Z_{T} V_{T}\right)^{-}<\infty$. The process $V_{t}=\sum_{u=0}^{t} \Delta V_{u}$ verifies $\Delta V_{u} \in-G_{u}$. Applying Proposition 3.3.2, [13], we get that

$$
\begin{aligned}
E\left(Z_{T} V_{T} \mid \mathcal{F}_{t}\right) & =V_{t} E\left(Z_{T} \mid \mathcal{F}_{t}\right)+E\left(Z_{T} \sum_{u=t+1}^{T} \Delta V_{u} \mid \mathcal{F}_{t}\right), \\
& =V_{t} Z_{t}+\sum_{u=t+1}^{T} E\left(Z_{u} \Delta V_{u} \mid \mathcal{F}_{t}\right) .
\end{aligned}
$$

Since $Z_{u} \Delta V_{u} \leq 0$, we deduce that $E\left(Z_{T} V_{T} \mid \mathcal{F}_{t}\right) \leq V_{t} Z_{t}$. Condition $\mathbf{S 2}$ 
naturally holds in the financial models with transaction costs. Indeed, if $\xi_{t} \in L^{\infty}\left(-G_{t}, \mathcal{F}_{t}\right)$ then $V_{u}=\xi_{t} I_{u>t}$ is a portfolio process whose only jump is $\Delta V_{t}=\xi_{t} \in-G_{t}$ and we have $\xi_{t}=V_{T}$.

For a given payoff $\xi \in L_{b}^{0}\left(\mathbf{R}^{d}\right)$ (i.e. bounded from below with respect to the partial ordering induced by $G_{T}$ ), we consider the convex set

$$
\Gamma_{\xi}:=\left\{x \in \mathbf{R}^{d}: \exists Y_{T} \in \mathcal{Y}_{0, b}^{T}(T) \text { s.t. } x+Y_{T} \geq_{G_{T}} \xi\right\}
$$

and the closed convex set

$$
D_{\xi}:=\left\{x \in \mathbf{R}^{d}: Z_{0} x \geq E Z_{T} \xi, \forall Z \in \mathcal{M}_{0}^{T}\left(G^{*}\right)\right\}
$$

We assume given a dual characterization of $\Gamma_{\xi}$ in section 3:

S3 : $\Gamma_{\xi}=D_{\xi}$.

This property is usually an important result, referred to as the "hedging theorem". It generally holds under some no-arbitrage conditions (see, e.g., [2], [1] and [4]).

3. Asymptotic Arbitrage via Consistent Price Systems. We fix a sequence $B^{n}:=\left(\Omega^{n}, \mathcal{F}^{n}, \mathbf{F}^{\mathbf{n}}=\left(\mathcal{F}_{t}^{n}\right)_{t \leq T}, P^{n}\right)$ of continuous-time stochastic basis verifying the usual conditions with $\mathcal{F}^{n}=\mathcal{F}_{T}^{n}$. The positive number $T$ is interpreted as a time horizon and may depend on $n$. We are given a pair of set-valued adapted processes $G^{n}=\left(G_{t}^{n}\right)_{t \leq T}$ and $G^{n *}=\left(G_{t}^{n *}\right)_{t \leq T}$ whose values are closed cones in $\mathbf{R}^{d}$ which are dual and define the corresponding models of Subsection 2.1. Recall that we assume that conditions S1 and S2 hold. For the sake of simplicity, we often omit the index $n$.

DEFINITION 3.1. A sequence of portfolios $\left(\widehat{V}^{n}\right)$ realizes an asymptotic arbitrage opportunity of the first kind if there exists a sequence $\left(x^{n}\right)$ such that the following holds for a subsequence:

3.1.a) $\widehat{V}_{T}^{n} \in x^{n}+\mathcal{Y}_{0, b}^{T}(T)$,

3.1.b) $\widehat{V}_{T}^{n} \in G_{T}$,

3.1.c) $x^{n} \rightarrow 0$,

3.1.d) $\lim _{n} P\left(\widehat{V}_{T}^{n} \geq_{G_{T}} \mathbf{1}\right)>0$.

imsart-aap ver. 2011/05/20 file: MafeLepinetteOstafe_02.04.tex date: April 3, 2012 
DeFinition 3.2. A sequence of portfolios $\left(\widehat{V}^{n}\right)$ realizes an asymptotic arbitrage opportunity of the second kind if there exists a subsequence satisfying:

3.2.a) $\widehat{V}_{T}^{n} \geq_{G_{T}}-\mathbf{1}$

3.2.b) $\left.\lim _{n} P\left(\widehat{V}_{T}^{n} \Varangle_{G_{T}}-\varepsilon \mathbf{1}\right)=0, \forall \varepsilon \in\right] 0,1[$,

3.2.c) there exists a bounded sequence of initial endowments $\left(x^{n} \mathbf{1}\right)$, with $x^{n} \in \mathbf{R}$, satisfying $\widehat{V}_{T}^{n} \in x^{n} \mathbf{1}+\mathcal{Y}_{0, b}^{T}(T)$ and $x^{\infty}:=\liminf _{n} x^{n}<0$.

REMARK 3.3. It is an easy exercise to notice that the definition of the asymptotic arbitrage of the second kind can be written equivalently if one considers in 3.2.c) a bounded sequence of initial endowments $x^{n} \in \mathbf{R}^{d}$ satisfying $\widehat{V}_{T}^{n} \in x^{n}+\mathcal{Y}_{0, b}^{T}(T)$, and such that $\liminf _{n} \max _{i \leq d} x^{n, i}<0$. Indeed, if $x^{n}$ is an initial endowment of $\widehat{V}_{T}^{n}$, then $y^{n}:=\left(\max _{i \leq d} x^{n, i}\right) \mathbf{1}$ is still an initial endowment for $\widehat{V}_{T}^{n}$.

In the same manner, we can equivalently define the asymptotic arbitrage of the first kind using a sequence of initial endowments of the form $\left(x^{n} \mathbf{1}\right)$, with $x^{n} \in \mathbf{R}$, but for our purposes it is more convenient to consider the definition with an initial endowment $x^{n} \in \mathbf{R}^{d}$.

Definition 3.4. A large security market $M=\left\{\left(B^{n}, G^{n}, T^{n}\right)\right\}$ has no asymptotic arbitrage opportunities of the first kind (respectively, of the second kind) if for any subsequence $(m)$ there are no trading strategies realizing an asymptotic arbitrage of the first kind (respectively, of the second kind) for $\left(B^{m}, G^{m}, T^{m}\right)$.

The goal of this section is to characterize the absence of asymptotic arbitrage opportunities of the first/second kind in terms of contiguity of a set of equivalent probability measures as defined below. To do so, we associate with every $Z \in \mathcal{M}_{0}^{T}\left(G^{*} \backslash\{0\}\right)$ the equivalent probability measure $d Q^{Z}:=\left(1 / Z_{0} \mathbf{1}\right) Z_{T} \mathbf{1} d P$ and we define the convex set

$$
\mathcal{Q}^{n}=\left\{Q^{Z}: Z \in \mathcal{M}_{0}^{T}\left(G^{*} \backslash\{0\}\right), Z_{0} \mathbf{1}=1\right\} .
$$

We assume that $\mathcal{Q}^{n}$ is not empty meaning that the No Free Lunch (NFL) condition holds, [3], for each model.

We then define the upper and lower envelopes of the measures of $\mathcal{Q}^{n}$ as follows:

$$
\overline{\mathcal{Q}}^{n}(A):=\sup _{Q \in \mathcal{Q}^{n}} Q(A), \quad \underline{\mathcal{Q}}^{n}(A):=\inf _{Q \in \mathcal{Q}^{n}} Q(A) .
$$

imsart-aap ver. 2011/05/20 file: MafeLepinetteOstafe_02.04.tex date: April 3, 2012 
Definition 3.5. A sequence of set-valued functions $\left(P^{n}\right)$ is contiguous with respect to the sequence of set-valued functions $\left(\overline{\mathcal{Q}}^{n}\right)$ (in symbols: $\left(P^{n}\right) \triangleleft$ $\left.\left(\overline{\mathcal{Q}}^{n}\right)\right)$ when the implication

$$
\lim _{n} \overline{\mathcal{Q}}^{n}\left(A^{n}\right)=0 \Rightarrow \lim _{n} P^{n}\left(A^{n}\right)=0
$$

holds for any sequence $A^{n} \in \mathcal{F}^{n}, n \geq 1$.

Now, we give the first results of this section:

Proposition 3.6. Assume that the Assumptions S1-S2-S3 hold. Then the following conditions are equivalent:

(a) there is no asymptotic arbitrage opportunity of the first kind (NAA1);

(b) $\left(P^{n}\right) \triangleleft\left(\overline{\mathcal{Q}}^{n}\right)$.

\section{Proof.}

- $(a) \Rightarrow(b)$. Suppose that there exists a sequence $A^{n} \in \mathcal{F}^{n}$ such that $\overline{\mathcal{Q}}^{n}\left(A^{n}\right)$ tends to 0 and $P\left(A^{n}\right) \rightarrow \alpha>0$. We consider $F^{n}=I_{A^{n}} \mathbf{1}$ as a contingent claim and $x^{n}=\overline{\mathcal{Q}}^{n}\left(A_{n}\right) \mathbf{1}$ as an initial endowment. For every $Z \in \mathcal{M}_{0}^{T}\left(G^{*} \backslash\{0\}\right)$, we have immediately by definition

$$
Z_{0} x^{n} \geq E Z_{T} F^{n}
$$

By virtue of Assumption S3, we deduce that $F^{n} \in x^{n}+\mathcal{Y}_{0, b}^{T}(T)$ so that the sequence $\left(F^{n}\right)$ realizes an asymptotic arbitrage opportunity of the first kind.

- $(b) \Rightarrow(a)$. Suppose that there exists a sequence $\left(\widehat{V}^{n}\right)$ realizing an asymptotic arbitrage opportunity of the first kind. Consider $Q \in \mathcal{Q}^{n}$ defined by $d Q=Z_{T} \mathbf{1} d P$. Then, according to Condition $\mathbf{S}_{1}$,

$$
0 \leq E Z_{T} \widehat{V}_{T}^{n} \leq Z_{0} x^{n} \leq\left|x^{n}\right|
$$

since $x^{n} \leq_{G_{0}}\left|x^{n}\right| \mathbf{1}$. Moreover,

$$
E Z_{T} \widehat{V}_{T}^{n} \geq E Z_{T} \widehat{V}_{T}^{n} I_{\widehat{V}_{T}^{n} \geq_{G_{T}} \mathbf{1}} \geq E Z_{T} \mathbf{1} I_{\widehat{V}_{T}^{n} \geq_{G_{T}} \mathbf{1}}=Q\left(\widehat{V}_{T}^{n} \geq_{G_{T}} \mathbf{1}\right) .
$$

It follows that

$$
\overline{\mathcal{Q}}^{n}\left(\widehat{V}_{T}^{n} \geq_{G_{T}} \mathbf{1}\right) \leq\left|x^{n}\right|
$$

and $\overline{\mathcal{Q}}^{n}\left(\widehat{V}_{T}^{n} \geq_{G_{T}} \mathbf{1}\right) \rightarrow 0$ which implies $P\left(\widehat{V}_{T}^{n} \geq_{G_{T}} \mathbf{1}\right) \rightarrow 0$ in contradiction with 3.1.d). 
REMARK 3.7. As shown in [12], the condition $(b)$ is equivalent to the following: (c) there exists a sequence $\left(R^{n}\right) \in \mathcal{Q}^{n}$ such that $\left(P^{n}\right) \triangleleft\left(R^{n}\right)$.

Proposition 3.8. Assume that the Assumptions S1-S2-S3 holds. Then the following conditions are equivalent:

(a) there is no asymptotic arbitrage opportunity of the second kind (NAA2);

(b) $\left(\underline{\mathcal{Q}}^{n}\right) \triangleleft\left(P^{n}\right)$.

\section{Proof.}

- $(a) \Rightarrow(b)$. Suppose that there exists a sequence $A^{n} \in \mathcal{F}^{n}$ such that $P^{n}\left(A^{n}\right)$ tends to 0 and $\mathcal{Q}^{n}\left(A^{n}\right) \rightarrow \alpha>0$. We define the contingent claim $F^{n}=-I_{A^{n}} 1$ we may interpret as the terminal value of a portfolio since it is replicable (e.g. by 0). Consider the bounded sequence of super-hedging prices for $F^{n}, y^{n}:=x^{n} \mathbf{1}:=-\underline{\mathcal{Q}}^{n}\left(A^{n}\right) \mathbf{1}$, i.e. $y^{n} \in \Gamma_{F^{n}}$. Indeed, for any $Z \in \mathcal{M}_{0}^{T}\left(G^{*} \backslash\{0\}\right)$ with $Z_{0} \mathbf{1}=1, Z_{0} y^{n}=-\underline{\mathcal{Q}}^{n}\left(A^{n}\right) \geq-Q^{Z}\left(A^{n}\right)=E Z_{T} F^{n}$ and we conclude using the Assumption S3. Since $\liminf _{n} x^{n}=x^{\infty}<0$, the sequence $\left(F^{n}\right)$ is an asymptotic arbitrage opportunity of the second kind.

- $(b) \Rightarrow(a)$. Suppose that there exists a sequence of portfolios $\left(\widehat{V}^{n}\right)$ realizing an asymptotic arbitrage opportunity of the second kind. Let us consider a sequence $\left(x^{n}\right)$ such that $\widehat{V}_{T}^{n} \in x^{n} \mathbf{1}+\mathcal{Y}_{0, b}^{T}(T)$ with $x^{\infty}:=\liminf _{n} x^{n}<0$. Under Assumption S3, for any $Z \in \mathcal{M}_{0}^{T}\left(G^{*} \backslash\{0\}\right)$ with $Z_{0} \mathbf{1}=1$, we have that

$$
\begin{aligned}
x^{n}=Z_{0} x^{n} \mathbf{1} & \geq E Z_{T} \widehat{V}_{T}^{n}=E Z_{T} \widehat{V}_{T}^{n} I_{\left\{\widehat{V}_{T}^{n} \geq-\varepsilon \mathbf{1}\right\}}+E Z_{T} \widehat{V}_{T}^{n} I_{\left\{\widehat{V}_{T}^{n} \ngtr-\varepsilon \mathbf{1}\right\}}, \\
x^{n} & \geq-\varepsilon Q^{Z}\left(\widehat{V}_{T}^{n} \geq-\varepsilon \mathbf{1}\right)-Q^{Z}\left(\widehat{V}_{T}^{n} \ngtr-\varepsilon \mathbf{1}\right), \\
& \geq-\varepsilon+(\varepsilon-1) Q^{Z}\left(\widehat{V}_{T}^{n} \ngtr-\varepsilon \mathbf{1}\right)
\end{aligned}
$$

where $\varepsilon \in(0,1)$ is arbitrarily chosen. Since the property 3.2.b) holds, the property $\left(\underline{\mathcal{Q}}^{n}\right) \triangleleft\left(P^{n}\right)$ implies that $\underline{\mathcal{Q}}^{n}\left(\widehat{V}_{T}^{n} \not=-\varepsilon \mathbf{1}\right) \rightarrow 0$. We choose, for each $n, Z^{n} \in \mathcal{M}_{0}^{T}\left(G^{*} \backslash\{0\}\right)$ such that $Q^{Z^{n}}\left(\widehat{V}_{T}^{n} \ngtr-\varepsilon \mathbf{1}\right) \leq \underline{\mathcal{Q}}^{n}\left(\widehat{V}_{T}^{n} \ngtr-\varepsilon \mathbf{1}\right)+n^{-1}$. From above, we deduce that $\liminf _{n} x^{n} \geq-\varepsilon$ whatever $\varepsilon \in(0,1)$ which yields a contradiction.

Definition 3.9. A sequence of portfolios $\left(\widehat{V}^{n}\right)$ realizes a strong asymptotic arbitrage opportunity of the first kind if there exists a sequence $\left(x^{n}\right)$ such that the following holds for a subsequence:

$$
\text { 3.9.a) } \widehat{V}_{T}^{n} \in x^{n}+\mathcal{Y}_{0, b}^{T}(T),
$$


3.9.b) $\widehat{V}_{T}^{n} \geq_{G_{T}} 0$,

3.9.c) $x^{n} \rightarrow 0$,

3.9.d) $\lim _{n} P\left(\widehat{V}_{T}^{n} \geq_{G_{T}} \mathbf{1}\right)=1$.

DEFINITION 3.10. A sequence of portfolios $\left(\widehat{V}^{n}\right)$ realizes a strong asymptotic arbitrage opportunity of the second kind if for a subsequence :

3.10.a) $\widehat{V}_{T}^{n} \geq_{G_{T}}-\mathbf{1}$,

3.10.b) $\left.\lim _{n} P\left(\widehat{V}_{T}^{n} \Varangle_{G_{T}}-\varepsilon \mathbf{1}\right)=0, \forall \varepsilon \in\right] 0,1[$,

3.10.c) there exists a bounded sequence of initial endowments $\left(x^{n} \mathbf{1}\right)$, with $x^{n} \in \mathbf{R}$, satisfying $\widehat{V}_{T}^{n} \in x^{n} \mathbf{1}+\mathcal{Y}_{0, b}^{T}(T)$ and $\liminf _{n} x^{n}=-1$.

Definition 3.11. A sequence of set-valued functions $\left(P^{n}\right)$ is (entirely) asymptotically separable from the sequence of set-valued functions $\left(\overline{\mathcal{Q}}^{n}\right)$, notation $\left(P^{n}\right) \triangle\left(\overline{\mathcal{Q}}^{n}\right)$, if there exists a subsequence $(m)$ with sets $A^{m} \in \mathcal{F}^{m}$ such that

$$
\lim _{m} \overline{\mathcal{Q}}^{m}\left(A^{m}\right)=0, \lim _{m} P^{m}\left(A^{m}\right)=1 .
$$

Proposition 3.12. Assume that S1-S2-S3 hold. Then the following conditions are equivalent:

(a) there is a strong asymptotic arbitrage opportunity of the first kind (SAA1);

(b) there is a strong asymptotic arbitrage opportunity of the second kind (SAA2);

(c) $\left(P^{n}\right) \triangle\left(\overline{\mathcal{Q}}^{n}\right)$;

(d) $\left(\underline{\mathcal{Q}}^{n}\right) \triangle\left(P^{n}\right)$.

Proof.

- $(a) \Rightarrow(c)$ Assume there exists a sequence of portfolios $\left(\widehat{V}^{n}\right)$ realizing a strong asymptotic arbitrage opportunity of the first kind. This means that there exists a subsequence $(m)$ such that

$$
\lim _{m} P^{m}\left(\widehat{V}_{T}^{m} \geq_{G_{T}} \mathbf{1}\right)=1, \quad \lim _{m} \widehat{V}_{0}^{m}=0 .
$$


Following the arguments of the proof of Proposition 3.6, the implication $(b) \Rightarrow(a)$, we obtain that $\lim _{m} \overline{\mathcal{Q}}^{m}\left(\widehat{V}_{T}^{m} \geq_{G_{T}} \mathbf{1}\right)=0$. We then take the sets $A^{m}:=\left\{\widehat{V}_{T}^{m} \geq_{G_{T}} \mathbf{1}\right\}$ for the separating sequence.

- $(c) \Rightarrow(a)$ Assume $\left(P^{n}\right) \triangle\left(\overline{\mathcal{Q}}^{n}\right)$. Then, there exists a sequence $(m)$ with sets $A^{m} \in \mathcal{F}^{m}$ such that

$$
\lim _{m} \overline{\mathcal{Q}}^{m}\left(A^{m}\right)=0, \lim _{m} P^{m}\left(A^{m}\right)=1 .
$$

Using the arguments in the proof of Proposition 3.6, the implication $(a) \Rightarrow$ (b), but with $\alpha=1$, we obtain a sequence of portfolios realizing a strong asymptotic arbitrage opportunity.

- $(a) \Rightarrow(b)$ Take any sequence $\left(\widehat{V}^{n}\right)$ realizing a strong asymptotic arbitrage opportunity of the first kind. We want to construct a sequence realizing a strong asymptotic arbitrage of the second kind. Define the sequence $\widehat{U}^{n}=-\mathbf{1}+\widehat{V}^{n}$. Using 3.9.b), we obtain that $\widehat{U}_{T}^{n} \geq_{G_{T}}-\mathbf{1}$, which is exactly the condition 3.10.a) of the definition of the asymptotic arbitrage opportunity of the second kind. We have

$$
P\left(\widehat{U}_{T}^{n} \nsupseteq G_{T}-\varepsilon \mathbf{1}\right)=1-P\left(\widehat{U}_{T}^{n} \geq_{G_{T}}-\varepsilon \mathbf{1}\right) \leq 1-P\left(\widehat{V}_{T}^{n} \geq_{G_{T}} \mathbf{1}\right) \rightarrow 0, n \rightarrow \infty
$$

which shows the condition 3.10.b). We only have to prove the condition 3.10.c). The condition 3.9.a) holds so that $\widehat{V}_{T}^{n} \in y^{n}+\mathcal{Y}_{0, b}^{T}(T)$ where $y^{n} \rightarrow 0$. We deduce that $\widehat{V}_{T}^{n} \in \alpha^{n} \mathbf{1}+\mathcal{Y}_{0, b}^{T}(T)$ where $\alpha^{n}:=\max _{i \leq d} y^{n i}$ and $\alpha^{n} \rightarrow 0$. It suffices to consider $x^{n}:=\alpha^{n}-1$ to conclude.

- $(b) \Rightarrow(a)$ Take any sequence $\left(\widehat{U}^{n}\right)$ realizing a strong asymptotic arbitrage opportunity of the second kind. We define a sequence realizing a strong asymptotic arbitrage opportunity of the first kind choosing the sequence $\widehat{V}^{n}=\widehat{U}^{n}+\mathbf{1}$. We only prove condition 3.9.c). It suffices to observe that

$$
P\left(\widehat{V}_{T}^{n} \nsucceq G_{T} \mathbf{1}\right)=P\left(\widehat{U}_{T}^{n} \nsucceq G_{T} 0\right) \leq \liminf _{\varepsilon \rightarrow 0, \varepsilon \in \mathbf{Q}^{+}} P\left(\widehat{U}_{T}^{n} \nsucceq G_{T}-\varepsilon \mathbf{1}\right)
$$

where $\mathbf{Q}^{+}$is the set of all strictly positive rational numbers. Taking any arbitrary $\delta>0$, we get that $P\left(\widehat{V}_{T}^{n} \Varangle_{G_{T}} \mathbf{1}\right) \leq \delta+P\left(\widehat{U}_{T}^{n} \Varangle_{G_{T}}-\varepsilon \mathbf{1}\right)$ for some $\varepsilon=\varepsilon(\delta)$. Using 3.10.b), we obtain $\lim _{n} P\left(\widehat{V}_{T}^{n} \Varangle_{G_{T}} \mathbf{1}\right) \leq \delta$ and then $\lim _{n} P\left(\widehat{V}_{T}^{n} \nsucceq G_{T} \mathbf{1}\right)=0$ as $\delta \rightarrow 0$.

4. Variant for markets with a numéraire. We consider markets whose first component of the price process $S$ is a numéraire (the cash $B$ ) in 
which the portfolios are liquidated. The asymptotic arbitrage opportunity concepts are defined similarly as in Section 3 but here we are concerned by the portfolios starting with an initial endowment expressed in cash and which are liquidated at the horizon date.

Definition 4.1. A sequence of portfolios $\left(\widehat{V}^{n}\right)$ realizes an asymptotic arbitrage of the first kind if there exists a sequence $\left(x^{n}\right) \in \mathbf{R}_{+}$such that for a subsequence:

$$
\begin{aligned}
& \text { 4.1.a) } \widehat{V}_{T}^{n} \in x^{n} e_{1}+\mathcal{Y}_{0, b}^{T}(T), \\
& \text { 4.1.b) } \widehat{V}_{T}^{n} \in G_{T} \text { a.s. } \\
& \text { 4.1.c) } x^{n} \rightarrow 0, \\
& \text { 4.1.d) } \lim _{n} P\left(\widehat{V}_{T}^{n} \geq_{G_{T}} e_{1}\right)>0 .
\end{aligned}
$$

Definition 4.2. A sequence of portfolios $\left(\widehat{V}^{n}\right)$ realizes an asymptotic arbitrage opportunity of the second kind if there exists a subsequence satisfying:

$$
\begin{aligned}
& \text { 4.2.a) } \widehat{V}_{T}^{n} \geq_{G_{T}}-e_{1}, \\
& \text { 4.2.b) } \left.\lim _{n} P\left(\widehat{V}_{T}^{n} \Varangle_{G_{T}}-\varepsilon e_{1}\right)=0, \forall \varepsilon \in\right] 0,1[,
\end{aligned}
$$

4.2.c) There exits a bounded sequence of initial endowments $\left(x^{n} e_{1}\right)$, with $x^{n} \in \mathbf{R}$, satisfying $\widehat{V}_{T}^{n} \in x^{n} e_{1}+\mathcal{Y}_{0, b}^{T}(T)$ and $x^{\infty}:=\liminf _{n} x^{n}<0$.

In this setting, we define for each $Z \in \mathcal{M}_{0}^{T}\left(G^{*} \backslash\{0\}\right), Q^{Z} \sim P$ such that

$$
\frac{d Q^{Z}}{d P}=\frac{Z_{T} e_{1}}{Z_{0} e_{1}}
$$

and we define the convex set:

$$
\mathcal{Q}^{n}=\left\{Q^{Z}, Z \in \mathcal{M}_{0}^{T}\left(G^{*} \backslash\{0\}\right), Z_{0} e_{1}=1\right\} .
$$

Notice that in the frictionless case, a consistent price system is a process having the form $Z_{t}=\rho_{t} S_{t}, \rho_{t} \in L^{0}\left(\mathbf{R}_{+}, \mathcal{F}_{t}\right)$. If $S^{(1)}=1$, i.e. the interest rate of the bond $r=0$, then $Z_{0} e_{1}=1$ means that $\rho$ is a density process or equivalently $d Q=Z_{T} e_{1} d P$ defines an equivalent martingale measure under which $S$ is a martingale. We may interpret our definition as an extension of that of [12]. Consider the upper and lower envelopes of the measures of $\mathcal{Q}^{n}$ as previously. We then obtain similar results under Conditions S1-S2-S3.

imsart-aap ver. 2011/05/20 file: MafeLepinetteOstafe_02.04.tex date: April 3, 2012 
Actually, the two approaches turn out to be equivalent under Condition (B) $(3.6 .3,[13]$ and [3]), and the additional hypothesis that the sequence (depending on $n$ through the cone and the horizon date)

$$
n \mapsto \min _{Z \in \mathcal{M}_{0}^{T}\left(G^{*} \backslash\{0\}\right), Z_{0} \mathbf{1}=1} Z_{0} e_{1}
$$

is bounded from below by a strictly positive constant (independent of $n$ ). Indeed, in this case, we can find $\alpha, \beta>0$ such that $\beta e_{1} \geq_{G_{0}} \mathbf{1} \geq G_{0} \alpha e_{1}$. It is then easy to construct an asymptotic arbitrage opportunity of the first kind (respectively of the second kind) following the former definition from an asymptotic arbitrage opportunity of the first kind (respectively of the second kind) according to the variant approach and vice-versa.

5. Examples. Throughout this section, we consider a continuous-time financial model with transaction costs defined as in [4], i.e. in the setting of the Kabanov and Campi-Schachermayer models. We assume that for each model the exchanges between assets are executed like in a "real world" where we go through the numéraire. To exchange some amount of the ithasset into the jth-asset, sell the ith-assets, get the money in cash (i.e. the bond) and buy jth-assets with this cash. This model is referred to as a model of stock market where the orders are either "buy a stock" or "sell a stock" (See Section 3.1.2, Examples 2 and 5, [13]). We model this assumption by the following:

RW: $\left(1+\lambda^{i, b}\right)\left(1+\lambda^{b, j}\right)=1+\lambda^{i, j}$ for every $i, j=0,1, \ldots, n$ and $i \neq j$.

5.1. One-stage APM by Ross. We study the example of [12] under the variant approach and under the $\mathbf{R W}$ condition. Recall that we are given a sequence of independent random variables $\left(\epsilon_{i}\right)_{i \geq 0}$ on a probability space $(\Omega, \mathcal{F}, P)$ taking values in a finite interval $[-N, N]$. We suppose that $E \epsilon_{i}=0, E \epsilon_{i}^{2}=1$. At time zero, asset prices are positive numbers $X_{0}^{i}, i \geq 0$. After a certain period (at time $T=1$ ), their positive discounted values are given by the following relations:

$$
\begin{aligned}
X_{1}^{0} & =X_{0}^{0}\left(1+\mu_{0}+\sigma_{0} \epsilon_{0}\right), \\
X_{1}^{i} & =X_{0}^{i}\left(1+\mu_{i}+\sigma_{i}\left(\gamma_{i} \epsilon_{0}+\bar{\gamma}_{i} \epsilon_{i}\right)\right), \quad i \geq 1 .
\end{aligned}
$$

The coefficients are here deterministic, $\sigma_{i}>0, \bar{\gamma}_{i}>0$ and $\gamma_{i}^{2}+\bar{\gamma}_{i}^{2}=1$, $\gamma_{0}=1$. The asset with number zero is interpreted as the market portfolio, $\gamma_{i}$ is the correlation coefficient between the rate of return for the market portfolio and the rate of return for the asset with number $i$. For $n \geq 1$, we

imsart-aap ver. 2011/05/20 file: MafeLepinetteOstafe_02.04.tex date: April 3, 2012 
consider the stochastic basis $\mathcal{B}^{n}:=\left(\Omega, \mathcal{F}^{n}, \mathbb{F}^{n}=\left(\mathcal{F}_{t}^{n}\right)_{t=0,1}, P^{n}\right)$ with the $(n+1)$-dimensional random process $S^{n}=\left(X_{t}^{0}, \ldots, X_{t}^{n}\right)_{t=0,1}$ where $\mathcal{F}_{0}^{n}$ is the trivial algebra, $\mathcal{F}_{1}^{n}=\mathcal{F}^{n}=\sigma\left(\epsilon_{0}, \ldots, \epsilon_{n}\right)$, and $P^{n}=P \mid \mathcal{F}^{n}$. We assume that the transaction costs coefficients of each model are constant and equal to $\lambda^{i}, i \geq 1$. They correspond to the exchanges from the risky assets number $i, i \geq 1$, to the bond (assumed to be constant and equal to 1), as well as from the bond to the risky assets. Moreover, we assume that there are no transaction costs regarding the exchanges between the bond and the portfolio market $X^{0}$, i.e. $\lambda^{0}:=0$. We suppose that there exists a constant $k$ such that

$$
\frac{1}{1-\lambda^{i}} \leq \frac{k}{1+\lambda^{i}}
$$

This assumption is not too restrictive from a practical point of view. For instance, if $\lambda^{i} \leq 0.5$ for all $i$, then $k=3$. More generally, the assumption means that there exits $\lambda^{*} \in(0,1)$ such that $\lambda^{i}<\lambda^{*}, \forall i$. The sequence $\mathcal{M}=\left\{\left(B^{n},\left(1, S^{n}\right), 1\right)\right\}$ is a large security market by our definition. We may rewrite the dynamics as in [12]:

$$
\begin{aligned}
X_{1}^{0} & =X_{0}^{0}\left(1+\sigma_{0}\left(\epsilon_{0}-b_{0}\right)\right), \\
X_{1}^{i} & =X_{0}^{i}\left(1+\sigma_{i} \gamma_{i}\left(\epsilon_{0}-b_{0}\right)+\sigma_{i} \bar{\gamma}_{i}\left(\epsilon_{i}-b_{i}\right)\right), \quad i \geq 1
\end{aligned}
$$

where

$$
b_{0}:=-\frac{\mu_{0}}{\sigma_{0}}, b_{i}:=\frac{\mu_{0} \beta_{i}-\mu_{i}}{\sigma_{i} \bar{\gamma}_{i}}, \quad \beta_{i}:=\gamma_{i} \sigma_{i} / \sigma_{0}, \quad i \geq 1 .
$$

Let $F_{i}$ be the distribution function of $\epsilon_{i}$. Put

$$
\underline{s}_{i}:=\inf \left\{t: F_{i}(t)>0\right\}, \quad \bar{s}_{i}:=\inf \left\{t: F_{i}(t)=1\right\},
$$

$\underline{d}_{i}:=b_{i}-\underline{s}_{i}, \bar{d}_{i}:=\bar{s}_{i}-b_{i}$, and $d_{i}^{0}:=\underline{d}_{i} \wedge \bar{d}_{i}$. As in [12], we suppose that $d_{i}^{0} \geq 0$. Moreover, let us define:

$$
d_{i}:=d_{i}^{0}+\frac{4 \lambda^{i}}{\left(1+\lambda^{i}\right) \sigma_{i} \bar{\gamma}_{i}}:=d_{i}^{0}+f_{i}, \quad i \geq 1, \quad d_{0}:=d_{0}^{0}:=d_{0}^{0}+f_{0} .
$$

As in [12], we suppose that each model has an equivalent probability measure so that there exists also a strictly consistent price system. In particular, we have $\left|b_{i}\right|<N$ and, without loss of generality, we assume that $N>1$.

Let us consider the following conditions:

C2: $\lim \sup _{i} \sqrt{b_{i}^{2}+\frac{2 \lambda^{i}}{\left(1-\lambda^{i}\right) \sigma_{i} \bar{\gamma}_{i}}}=0$.

P2: $\lim \sup _{i}\left(\left|b_{i}\right|-2 \frac{\lambda^{i}}{\left(1-\lambda^{i}\right) \sigma_{i} \bar{\gamma}_{i}}\right) \leq 0$ and $\lim \sup _{i} \frac{\lambda^{i}}{\left(1-\lambda^{i}\right) \sigma_{i} \bar{\gamma}_{i}} \in(0, \infty)$.

imsart-aap ver. 2011/05/20 file: MafeLepinetteOstafe_02.04.tex date: April 3, 2012 
Proposition 5.1. The following statements hold:

(a) $\inf _{i} d_{i}=0 \Leftrightarrow S A A 1$

(b) $\inf _{i} d_{i}>0 \Leftrightarrow N A A 1$;

(c) $\mathbf{C 2}$ or $\mathbf{P 2} \Leftrightarrow N A A 2$.

Proof. Under Condition RW, we may assume without loss of generality that the only exchanges occur between the bond and the risky assets, i.e. there is no exchange between two risky assets. Recall that, in this model, there are no transaction costs between the bond and the portfolio market. Then, the terminal value of a portfolio, once liquidated, can be expressed as follows:

$$
V_{1}^{n}=x^{n}+\sum_{i=0}^{n} \phi_{i}\left(X_{1}^{i}-X_{0}^{i}\right)-\sum_{i=1}^{n} \lambda^{i}\left|\phi_{i}\right|\left(X_{0}^{i}+X_{1}^{i}\right)
$$

where $\left(\phi_{i}\right)_{i=0, \ldots, n}$ is the composition of the portfolio at date zero in the risky assets and $x^{n}$ is the initial endowment expressed in the bond. The first two terms of $V_{1}^{n}$ represent respectively the initial endowment and the variations of the portfolio due to the price movements. The last one corresponds respectively to the transaction costs that have to be paid due to the passage from $x^{n}$ to $\phi$ and to the liquidation of the portfolio at date 1 . We use the notations of [12]:

$$
a_{0}:=\sum_{i=0}^{n} \phi_{i} X_{0}^{i} \sigma_{i} \gamma_{i}, \quad a_{i}:=\phi_{i} X_{0}^{i} \sigma_{i} \bar{\gamma}_{i}, \quad i \geq 1 .
$$

The terminal value of the portfolio can be rewritten as:

$$
\begin{aligned}
V_{1}^{n} & =x^{n}+\sum_{i=0}^{n} a_{i}\left(\epsilon_{i}-b_{i}\right)-\sum_{i=1}^{n}\left|\phi_{i}\right| \lambda^{i}\left(X_{0}^{i}+X_{1}^{i}\right) \\
& =x^{n}+\sum_{i=0}^{n} a_{i}\left(\epsilon_{i}-b_{i}\right)-2 \sum_{i=1}^{n} \lambda^{i}\left|\phi_{i}\right| X_{0}^{i}-\sum_{i=1}^{n} \lambda^{i}\left|\phi_{i}\right|\left(X_{1}^{i}-X_{0}^{i}\right) \\
& =x^{n}+\sum_{i=0}^{n} \alpha_{i}\left(\epsilon_{i}-b_{i}\right)-2 \sum_{i=1}^{n} \lambda^{i} \frac{\left|a_{i}\right|}{\sigma_{i} \bar{\gamma}_{i}}
\end{aligned}
$$

where

$$
\alpha_{0}:=a_{0}-\sum_{i=1}^{n} \lambda^{i}\left|a_{i}\right| \frac{\gamma_{i}}{\bar{\gamma}_{i}}, \quad \alpha_{i}:=a_{i}-\lambda^{i}\left|a_{i}\right|, \quad i \geq 1 .
$$

imsart-aap ver. 2011/05/20 file: MafeLepinetteOstafe_02.04.tex date: April 3, 2012 
Note that, for $i \geq 1, a_{i}=\alpha_{i} /\left(1-\lambda^{i}\right)$ if $\alpha_{i} \geq 0$ and $a_{i}=\alpha_{i} /\left(1+\lambda^{i}\right)$ if $\alpha_{i} \leq 0$ so that $\left(\alpha_{i}\right)_{i=0, \ldots, n}$ are uniquely determined and vice-versa.

- Assume that $\inf _{i} d_{i}=0$. Then, there exists a subsequence $\left(i_{k}\right)$ such that $d_{i}^{0}+f_{i}<2^{-i}$. We then construct a strong asymptotic arbitrage opportunity only using the risky assets corresponding to this subsequence. We follow the proof of [12]. We set $\alpha_{i}^{2 n}:=1_{\bar{\Gamma} \cap\{i \geq n+1\}}-1_{\Gamma \cap\{i \geq n+1\}}, i \geq n+1$, where $\Gamma:=\left\{i: \bar{d}_{i}<\underline{d}_{i}\right\}$ and $\bar{\Gamma}$ is the complementary of $\Gamma$. Note that there is an abuse of notation as in [12]. The number $2 n$ means that we work with the model in which we consider the $2 n$ assets whose indices belong to the subsequence $\left(i_{k}\right)$. In other words we only trade the assets having the same indices than the subsequence. As in [12], but taking $x^{2 n}:=2^{-n}(1+k)$, we deduce that

$$
\begin{aligned}
V_{1}^{2 n} & \geq \sum_{i=n+1}^{2 n}\left(\left(\bar{s}_{i}-\epsilon_{i}\right) 1_{\Gamma}+\left(\epsilon_{i}-\underline{s}_{i}\right) 1_{\bar{\Gamma}}\right)+2^{-n}-\sum_{i=n+1}^{2 n}\left(\bar{d}_{i} 1_{\Gamma}+\underline{d}_{i} 1_{\bar{\Gamma}}\right) \\
& +k 2^{-n}-2 \sum_{i=n+1}^{2 n} \lambda^{i} \frac{1}{\left(1-\lambda^{i} \operatorname{sign}\left(\alpha_{i}\right)\right) \sigma_{i} \bar{\gamma}_{i}} .
\end{aligned}
$$

Observe that

$\lambda^{i} \frac{1}{\left(1-\lambda^{i} \operatorname{sign}\left(\alpha_{i}\right)\right) \sigma_{i} \bar{\gamma}_{i}} \leq \lambda^{i} \frac{1}{\left(1-\lambda^{i}\right) \sigma_{i} \bar{\gamma}_{i}} \leq \lambda^{i} \frac{k}{\left(1+\lambda^{i}\right) \sigma_{i} \bar{\gamma}_{i}} \leq k f_{i} / 4 \leq k 2^{-i} / 4$.

It follows that

$$
V_{1}^{2 n} \geq \sum_{i=n+1}^{2 n}\left(\left(\bar{s}_{i}-\epsilon_{i}\right) 1_{\Gamma}+\left(\epsilon_{i}-\underline{s}_{i}\right) 1_{\bar{\Gamma}}\right)
$$

and we conclude like in [12] that $V_{1}^{2 n}$ converges a.s. to $\infty$ as $n \rightarrow \infty$, i.e. there is a strong asymptotic arbitrage opportunity of the first kind.

- Assume that $\inf _{i} d_{i}=\delta>0$. Then, using a similar argument like in [12], we have the following inequalities on a non-null set: 


$$
\begin{aligned}
V_{1}^{n} & \leq x^{n}-\sum_{i=0}^{n}\left|\alpha_{i}\right| \frac{d_{i}^{0}}{2}-2 \sum_{i=1}^{n} \lambda^{i} \frac{\left|a_{i}\right|}{\sigma_{i} \bar{\gamma}_{i}} \\
& \leq x^{n}-\sum_{i=0}^{n}\left|\alpha_{i}\right| \frac{d_{i}}{2}+\sum_{i=1}^{n}\left|\alpha_{i}\right| \frac{f_{i}}{2}-2 \sum_{i=1}^{n} \lambda^{i} \frac{\left|\alpha_{i}\right|}{\left(1-\lambda^{i} \operatorname{sign}\left(\alpha_{i}\right)\right) \sigma_{i} \bar{\gamma}_{i}} \\
& \leq x^{n}-\sum_{i=0}^{n}\left|\alpha_{i}\right| \frac{d_{i}}{2}+\sum_{i=1}^{n}\left|\alpha_{i}\right| \frac{f_{i}}{2}-2 \sum_{i=1}^{n} \lambda^{i} \frac{\left|\alpha_{i}\right|}{\left(1+\lambda^{i}\right) \sigma_{i} \bar{\gamma}_{i}} \\
& \leq x^{n}-\sum_{i=0}^{n}\left|\alpha_{i}\right| \frac{d_{i}}{2}+\sum_{i=1}^{n} \frac{\left|\alpha_{i}\right|}{2}\left(f_{i}-4 \lambda^{i} \frac{\left|\alpha_{i}\right|}{\left(1+\lambda^{i}\right) \sigma_{i} \bar{\gamma}_{i}}\right) \\
& \leq x^{n}-\sum_{i=0}^{n}\left|\alpha_{i}\right| \frac{d_{i}}{2} \leq x^{n}-\frac{\delta}{2} \sum_{i=0}^{n}\left|\alpha_{i}\right| .
\end{aligned}
$$

With $V_{1}^{n} \geq 0$ and $x^{n} \rightarrow 0$, it follows that $\sum_{i=0}^{n}\left|\alpha_{i}\right| \rightarrow 0$ as $n \rightarrow \infty$. From the inequality

$$
0 \leq V_{1}^{n} \leq x^{n}+2 N \sum_{i=0}^{n}\left|\alpha_{i}\right|
$$

we deduce that $V_{1}^{n} \rightarrow 0$ as $n \rightarrow \infty$. Hence, there is no strong asymptotic arbitrage opportunity of the first kind. We then conclude about $(a)$ and also about $(b)$ as a consequence.

Let us now prove Statement (c).

- Let us first assume that (NAA2) holds and $\lim \sup _{i} \tilde{b}_{i}>0$ where

$$
\tilde{b}_{i}:=\sqrt{b_{i}^{2}+\frac{2 \lambda^{i}}{\left(1-\lambda^{i}\right) \sigma_{i} \bar{\gamma}_{i}}} .
$$

Let us also suppose that Condition P2 does not hold. Under the conditions above, we show that it is possible to construct an asymptotic arbitrage opportunity of the second kind hence a contradiction. We may assume without loss of generality that $\nu:=\inf _{i} \tilde{b}_{i}>0$. Since $N\left|b_{i}\right| \geq b_{i}^{2}$ we get that

$$
N\left|b_{i}\right|+N \frac{2 \lambda^{i}}{\left(1-\lambda^{i}\right) \sigma_{i} \bar{\gamma}_{i}} \geq b_{i}^{2}+\frac{2 \lambda^{i}}{\left(1-\lambda^{i}\right) \sigma_{i} \bar{\gamma}_{i}} .
$$

From there, we may assume that we also have

$$
\left|b_{i}\right|+\frac{2 \lambda^{i}}{\left(1-\lambda^{i}\right) \sigma_{i} \bar{\gamma}_{i}}>\widetilde{\nu}
$$


where $\widetilde{\nu}>0$ is a constant. Let us denote $D_{n}^{2}:=\sum_{i=0}^{n} \tilde{b}_{i}^{2}$ and consider a terminal portfolio value:

$$
V_{1}^{n}:=x^{n}+\sum_{i=0}^{n} \alpha_{i}\left(\epsilon_{i}-b_{i}\right)-2 \sum_{i=1}^{n} \lambda^{i} \frac{\left|\alpha_{i}\right|}{\left(1-\operatorname{sign}\left(\alpha_{i}\right) \lambda^{i}\right) \sigma_{i} \bar{\gamma}_{i}} .
$$

The idea is to choose the coefficients $\alpha_{i}=\alpha_{i}^{n}$ so that $V_{1}^{n} \rightarrow 0$ a.s. and

$$
x^{n}=\sum_{i=0}^{n} \alpha_{i} b_{i}+2 \sum_{i=1}^{n} \lambda^{i} \frac{\left|\alpha_{i}\right|}{\left(1-\operatorname{sign}\left(\alpha_{i}\right) \lambda^{i}\right) \sigma_{i} \bar{\gamma}_{i}} .
$$

It follows that

$$
V_{1}^{n}=\sum_{i=0}^{n} \alpha_{i} \epsilon_{i}
$$

Renormalizing the sequence $\left(V_{1}^{n}\right)$ if necessary, we deduce that $\left|V_{1}^{n}\right| \leq 1$ and applying the strong law of large numbers, we shall conclude that $V_{1}^{n} \rightarrow 0$ a.s. It remains to construct the coefficients $\left(\alpha_{i}\right)$ and to show that $\lim \inf x^{n}<0$. We put

$$
e_{i}:=\quad b_{i}-2 \frac{\lambda^{i}}{\left(1+\lambda^{i}\right) \sigma_{i} \bar{\gamma}_{i}}
$$

- First Case. We suppose there exists $c>0$ and a subsequence such that

$$
e_{i} \geq c \tilde{b}_{i}
$$

We choose $\alpha_{i}:=-\frac{\nu^{2} \tilde{b}_{i}}{N^{2} D_{n}^{2}}$ so that $\left|V_{1}^{n}\right| \leq 1$. Moreover, the inequality

$$
x_{n}=-\frac{\nu^{2}}{N^{2} D_{n}^{2}} \sum_{i=0}^{n} \tilde{b}_{i} e_{i} \leq-\frac{c \nu^{2}}{N^{2}}
$$

implies that $\lim \inf x_{n} \in(-\infty, 0)$. Since $D_{n} \geq C n$ where $C>0$, we deduce that $V_{1}^{n} \rightarrow 0$ by virtue of the strong law of large numbers.

-Second Case. We suppose that $e_{i} \leq 0$. Since Condition P2 does not hold, either $(i): \lim \sup _{i}\left(\left|b_{i}\right|-2 \frac{\lambda^{i}}{\left(1-\lambda_{i}\right) \sigma_{i} \bar{\gamma}_{i}}\right)>0$ or $(i i): \lim \sup _{i} \frac{\lambda^{i}}{\left(1-\lambda^{i}\right) \sigma_{i} \bar{\gamma}_{i}}=0$. In the second case $(i i)$, we then deduce that $\lim \sup _{i} b_{i}=0$ if the condition $(i)$ is not satisfied hence a contradiction. Then, we may assume that there exists a constant $c \in(0,1)$ such that

$$
\left(\left|b_{i}\right|-2 \frac{\lambda^{i}}{\left(1-\lambda^{i}\right) \sigma_{i} \bar{\gamma}_{i}}\right) \geq c \sqrt{b_{i}^{2}+\frac{2 \lambda^{i}}{\left(1-\lambda^{i}\right) \sigma_{i} \bar{\gamma}_{i}}} .
$$

imsart-aap ver. 2011/05/20 file: MafeLepinetteOstafe_02.04.tex date: April 3, 2012 
Indeed, the second term in the rhs of the inequality above turns out to be bounded (for a subsequence) by virtue of $(i)$. From now on, consider the portfolio terminal value:

$$
V_{1}^{n}:=-\frac{\nu}{N D_{n}} \sum_{i=1}^{n} \operatorname{sign}\left(b_{i}\right) \sqrt{b_{i}^{2}+\frac{2 \lambda^{i}}{\left(1-\lambda^{i}\right) \sigma_{i} \bar{\gamma}_{i}}} \epsilon_{i}-\frac{\nu}{N D_{n}} \epsilon_{0} .
$$

It satisfies $\left|V_{1}^{n}\right| \leq 1$ and by virtue of the Bienaymé-Tchebychev inequality,

$$
P\left(\left|V_{1}^{n}\right| \geq \varepsilon\right) \leq \frac{\nu^{2}}{N^{2} D_{n} \varepsilon^{2}} \rightarrow 0, \quad n \rightarrow \infty
$$

since $D_{n} \geq \nu n$. At last, recall that the random variables $\left(\epsilon_{i}\right)_{i \geq 0}$ are independent and identically distributed under the initial probability measure. We deduce that $V_{1}^{n}$ is the terminal value of a portfolio of the form (5.4) if and only if

$$
\alpha_{i}=-\operatorname{sign}\left(b_{i}\right) \frac{\nu}{N D_{n}} \sqrt{b_{i}^{2}+\frac{2 \lambda^{i}}{\left(1-\lambda^{i}\right) \sigma_{i} \bar{\gamma}_{i}}}, \quad i \geq 1, \quad \alpha_{0}=-\frac{\nu}{N D_{n}} b_{0} .
$$

We deduce that

$$
x^{n}=-\frac{\nu}{N D_{n}} b_{0}^{2}-\frac{\nu}{N D_{n}} \sum_{i=1}^{n} \sqrt{b_{i}^{2}+\frac{2 \lambda^{i}}{\left(1-\lambda^{i}\right) \sigma_{i} \bar{\gamma}_{i}}}\left(\left|b_{i}\right|-\frac{2 \lambda^{i}}{\left(1-\lambda^{i} \operatorname{sign}\left(\alpha_{i}\right)\right) \sigma_{i} \bar{\gamma}_{i}}\right) .
$$

We then deduce that $x^{n} \leq-\frac{c \nu}{N}$ and we conclude that $\left(V_{1}^{n}\right)$ realizes an asymptotic arbitrage opportunity of the second kind.

- Let us suppose that

$$
\limsup _{i} \sqrt{b_{i}^{2}+\frac{2 \lambda^{i}}{\left(1-\lambda^{i}\right) \sigma_{i} \bar{\gamma}_{i}}}=0 .
$$

It follows that $\lim \sup _{i}\left|b_{i}\right|=0$. Following the reasoning of [12], we deduce that $\lim \sup _{i} d_{i}^{0} \geq C$, where $C$ is a strictly positive such that $\underline{s}_{i} \leq-C$ and $\bar{s}_{i} \geq C$, and $\delta:=\inf _{i} d_{i}^{0}>0$. We also deduce that

$$
\limsup _{i} \frac{2 \lambda^{i}}{\left(1+\lambda^{i}\right) \sigma_{i} \bar{\gamma}_{i}}=0 .
$$

We may assume without loss of generality that

$$
\sup _{i}\left(\frac{2 \lambda^{i}}{\left(1+\lambda^{i}\right) \sigma_{i} \bar{\gamma}_{i}}\right) \leq \frac{\delta}{4}
$$

imsart-aap ver. 2011/05/20 file: MafeLepinetteOstafe_02.04.tex date: April 3, 2012 
We deduce the existence of $\tilde{\delta}>0$ such that

$$
\inf _{i}\left(\frac{d_{i}^{0}}{2}+\frac{2 \lambda^{i}}{\left(1+\lambda^{i}\right) \sigma_{i} \bar{\gamma}_{i}}\right)>\tilde{\delta}
$$

Let $\left(x^{n}, \alpha^{n}\right)$ be a sequence such that the properties (3.a) and (3.c) of a strategy realizing (AA2) are fulfilled, i.e. $x^{n} \rightarrow-x<0$ and

$$
-V_{1}^{n}=-x^{n}-\sum_{i=0}^{n} \alpha_{i}\left(\epsilon_{i}-b_{i}\right)+2 \sum_{i=1}^{n} \lambda^{i} \frac{\left|a_{i}\right|}{\sigma_{i} \bar{\gamma}_{i}} \leq 1 .
$$

Then, on a non-null set, we deduce that

$$
\begin{array}{r}
-x^{n}+\sum_{i=0}^{n}\left|\alpha_{i}\right| \frac{d_{i}^{0}}{2}+2 \sum_{i=1}^{n} \lambda^{i} \frac{\left|\alpha_{i}\right|}{\left(1-\lambda^{i} \operatorname{sign}\left(\alpha_{i}\right)\right) \sigma_{i} \bar{\gamma}_{i}} \leq 1 \\
-x^{n}+\sum_{i=0}^{n}\left|\alpha_{i}\right|\left(\frac{d_{i}^{0}}{2}+\frac{2 \lambda^{i}}{\left(1+\lambda^{i}\right) \sigma_{i} \bar{\gamma}_{i}}\right) \leq 1
\end{array}
$$

Then, with $n$ large enough and $\gamma:=x / 2$, we have $\gamma+\tilde{\delta} \sum_{i=0}^{n}\left|\alpha_{i}\right| \leq 1$ and $\sum_{i=0}^{n}\left|\alpha_{i}\right| \leq(1-\gamma) / \tilde{\delta}$. Observe that we can also choose $\tilde{\delta}$ smaller so that the last inequality holds for all $n$. Since

$$
\limsup _{i}\left(\left|b_{i}\right|+\frac{2 \lambda^{i}}{\left(1+\lambda^{i}\right) \sigma_{i} \bar{\gamma}_{i}}\right)=0
$$

we also may assume that

$$
\sup _{i}\left(\left|b_{i}\right|-\frac{2 \lambda^{i}}{\left(1+\lambda^{i}\right) \sigma_{i} \bar{\gamma}_{i}}\right) \leq \frac{\tilde{\delta} \gamma}{2(1-\gamma)} .
$$

We deduce that, with $n$ large enough,

$$
\begin{aligned}
-V_{1}^{n} & \geq \gamma+\sum_{i=0}^{n} \alpha_{i} \epsilon_{i}-\sum_{i=0}^{n}\left|\alpha_{i}\right|\left(\left|b_{i}\right|-\frac{2 \lambda^{i}}{\left(1+\lambda^{i}\right) \sigma_{i} \bar{\gamma}_{i}}\right) \\
& \geq \frac{\gamma}{2}+\sum_{i=0}^{n} \alpha_{i} \epsilon_{i} .
\end{aligned}
$$

We conclude that for $n$ large enough,

$$
\begin{aligned}
P\left(V_{1}^{n} \leq-\gamma / 4\right)=P\left(-V_{1}^{n} \geq \gamma / 4\right) & \geq E\left(-V_{1}^{n}-\gamma / 4\right)^{+} \wedge 1 \\
& \geq E\left(-V_{1}^{n}-\gamma / 4\right) \wedge 1 \\
& \geq E\left(-V_{1}^{n}-\gamma / 4\right) \geq \gamma / 4
\end{aligned}
$$

hence ( $N A A 2)$ holds. Under the condition P2, we do the same reasoning since the inequalities (5.5) and (5.6) remains valid.

imsart-aap ver. 2011/05/20 file: MafeLepinetteOstafe_02.04.tex date: April 3, 2012 
5.2. The large Black-Scholes market. We consider the large Black and Scholes market example of Kabanov and Kramkov [12]. We are given a sequence of markets whose horizon dates are $T^{n}=T$ for all $n \geq 1$. Let $\left(\Omega, \mathcal{F}, \mathbf{F}=\left(\mathcal{F}_{t}\right)_{t \leq T}, P\right)$ be a stochastic basis with a countable set of independent one-dimensional standard Brownian motions $\left(W^{i}\right)_{i \geq 0}$. We define $B^{n}=\left(W^{0}, \ldots, W^{n}\right)$, and let $\mathbf{G}^{n}=\left(\mathcal{G}_{t}^{n}\right)$ be a subfiltration of $\mathbf{F}$ such that $\left(B^{n}, \mathbf{G}^{n}\right)$ is a $(n+1)$-dimensional standard Wiener process. The behaviour of the stock prices is described as follows:

$$
\begin{aligned}
d X_{t}^{0} & =\mu_{t}^{0} X_{t}^{0} d t+\sigma_{t}^{0} X_{t}^{0} d W_{t}^{0} \\
d X_{t}^{i} & =\mu_{t}^{i} X_{t}^{i} d t+\sigma_{t}^{i} X_{t}^{i}\left(\gamma_{t}^{i} d W_{t}^{0}+\bar{\gamma}_{t}^{i} d W_{t}^{i}\right), \quad i \in \mathrm{N}
\end{aligned}
$$

with deterministic (strictly positive) initial points. The coefficients are $\mathbf{G}^{i}$ predictable processes verifying

$$
\int_{0}^{T}\left|\mu_{s}^{i}\right|^{2} d s<\infty, \quad \int_{0}^{T}\left|\sigma_{s}^{i}\right|^{2} d s<\infty
$$

and $\left|\gamma_{t}^{i}\right|^{2}+\left|\bar{\gamma}_{t}^{i}\right|^{2}=1$. To avoid degeneracy we shall assume that $\sigma^{i}>0$ and $\bar{\gamma}^{i}>0$. Moreover, we assume that there exists a bond $B_{t}=1$ for all $t \geq 0$.

We shall study the absence of asymptotic arbitrage opportunities of the first kind according to the variant definition of Section 4. Observe that in our example $\mathcal{Y}_{0, b}^{T, \infty}(T)$ is Fatou-closed, [4], since the price process provides a strictly consistent price system. We want to characterize the probability measures $Q^{n} \in \mathcal{Q}^{n}$, i.e. the probability measures $Q \sim P$ such that $\frac{d Q}{d P}=$ $Z_{T} e_{1}$ where $Z_{T} \in \mathcal{M}_{0}^{T}\left(G^{*} \backslash\{0\}, P\right)$ and $Z_{0} e_{1}=1$. To do so, we first describe the consistent price systems. Let us denote by $\lambda_{t}^{i, b}, \lambda_{t}^{b, i}$, for $t \geq 0$, and $i=0, \ldots, n$, the transaction costs coefficients characterizing the exchange between the risky assets and the bond. We assume that $\lambda_{t}^{i, b}>0$ and $\lambda_{t}^{b, i}>0$ for all $i=0, \ldots, n$.

Definition 5.2. We say that the process $Y \in \mathbf{R}_{+}^{n+1}$ is a $\lambda$-consistent price system for the prices $\left(X^{i}\right)_{i \leq n}$ if there exists $Q \sim P$ such that $Y$ is a $Q$-martingale and

$$
\frac{X_{t}^{i}}{1+\lambda_{t}^{i, b}} \leq Y_{t}^{i} \leq\left(1+\lambda_{t}^{b, i}\right) X_{t}^{i}, \quad i=0, \ldots, n .
$$

Lemma 5.3. Assume that Assumption RW holds. Then, there exists a consistent price system $Z \in \mathcal{M}_{0}^{T}\left(G^{*} \backslash\{0\}\right)$ if and only if there is a $\lambda$ consistent price system for the prices $\left(X^{i}\right)_{i \leq n}$.

imsart-aap ver. 2011/05/20 file: MafeLepinetteOstafe_02.04.tex date: April 3, 2012 
Proof. • " $\Rightarrow "$ Assume that there exists a consistent price system $Z$ in $\mathcal{M}_{0}^{T}\left(G^{*} \backslash\{0\}\right)$, i.e. $Z$ is a martingale and $Z_{t} \in G_{t}^{*} \backslash\{0\}$, for all $t \leq T$. Recall that $G^{*}$ is the $(n+2)$-dimensional cone defined by the transaction costs $\lambda^{i, b}$ and $\lambda^{b, i}$ for $i \leq n$. Denoting $Z=\left(Z^{b}, Z^{0}, \cdots, Z^{n}\right)$, we interpret $Z^{b}$ as a numéraire and take $Y$ defined as follows:

$$
Y_{t}:=\left(\frac{Z_{t}^{0}}{Z_{t}^{b}}, \ldots, \frac{Z_{t}^{n}}{Z_{t}^{b}}\right) .
$$

Define $Q$ such that $d Q / d P=Z_{T}^{b} / Z_{0}^{b}$. Since $\left(Z_{t}\right)_{t \leq T}$ is a martingale, it is clear that $Y$ is a $Q$-martingale. In order for $Y$ to be a $\lambda$-consistent price system, we only have to prove (5.7) but these inequalities follow immediately from the fact that $Z_{t} \in G_{t}^{*} \backslash\{0\}$, for all $t \leq T$ (see the definition of $G^{*}$ in [13]).

- " $\Leftarrow$ " Assume that $Y$ is a $\lambda$-consistent price system, i.e. there exists a probability measure $Q \sim P$ such that $Y$ is a $Q$-martingale and the inequalities (5.7) hold. Then we define $\rho_{t}^{0}$ by $\rho_{t}^{0}:=E\left[d Q / d P \mid \mathcal{F}_{t}\right]$ and $Z_{t}^{j}$ by $Z_{t}^{j}:=Y_{t}^{j} \rho_{t}^{0}$ for every $j=0, \ldots, n, Z_{t}^{b}:=\rho_{t}^{0}$. Now, it is easily seen that, since $\mathrm{Y}$ is a $Q$-martingale, $Z=\left(Z^{b}, Z^{0}, \ldots, Z^{n}\right)$ is a $P$-martingale. The proof is now completed because the inequalities (5.7) imply the fact that $Z$ lies in $G^{*} \backslash\{0\}$ under Assumption RW.

From there, we deduce that for each model,

$$
\overline{\mathcal{Q}}^{n}(A)=\sup _{Q \in \mathcal{Q}^{n}} Q(A)=\widetilde{\mathcal{Q}}^{n}(A):=\sup _{Q \in \widetilde{Q}^{n}} Q(A)
$$

where

$$
\widetilde{Q}^{n}:=\left\{Q: d Q=\rho_{T} d P, \rho \in \widetilde{M}_{e}\right\}
$$

and $\widetilde{M}_{e}$ is the set of all density processes such that there exists a $\lambda$-consistent price system for the prices $\left(X^{i}\right)_{i \leq n}$ under the probability measure defined by $d Q=\rho_{T} d P$. From now on, let us denote for a given $\lambda$-consistent price system $Y^{n}$ of the $n$-th model,

$$
\widetilde{Q}\left(Y^{n}\right):=\left\{Q: d Q=\rho_{T} d P, \quad \rho \in \widetilde{M}_{e}\left(Y^{n}\right)\right\}
$$

and $\widetilde{M}_{e}\left(Y^{n}\right)$ is the set of all density processes such that the $\lambda$-consistent price system $Y^{n}$ is a martingale under the probability measure defined by $d Q=\rho_{T} d P$. Notice that $\widetilde{M}_{e}$ is the union of all $\widetilde{M}_{e}\left(Y^{n}\right)$. We then denote by $\widetilde{\widetilde{Q}\left(Y^{n}\right)}$ the upper envelope of the probability measures of $\widetilde{Q}\left(Y^{n}\right)$. For our next purpose we remind Proposition 3.6 above in its variant version.

imsart-aap ver. 2011/05/20 file: MafeLepinetteOstafe_02.04.tex date: April 3, 2012 
Proposition 5.4. Assume that each model is defined by the matrixvalued transaction costs process $\left(\lambda^{i, j}\right)_{i, j \in\{b, 0, \cdots, n\}}$ verifying Condition RW. Then, the following conditions are equivalent:

(a) there is no asymptotic arbitrage of the first kind (NAA1),

(b) $\left(P^{n}\right) \triangleleft\left(\overline{\widetilde{\mathcal{Q}}^{n}}\right)$,

(c) there exists a sequence $\left(Y^{n}\right)$ of $\lambda$-consistent price systems such that

$$
\left(P^{n}\right) \triangleleft\left(\overline{\widetilde{\mathcal{Q}}^{n}\left(Y^{n}\right)}\right),
$$

The main result of this example is the following.

Proposition 5.5. Assume that the transaction costs coefficients are constant in time and strictly positive. Suppose that the coefficients $\mu_{i}, \sigma_{i}, \gamma_{i}, \bar{\gamma}_{i}$ are deterministic. Then, there is no asymptotic arbitrage of the first kind (NAA1).

Proof. It suffices to check Property (c) of Proposition 5.4. To do so, we construct, for each $n$ and for each $i=0, \cdots, n$, an $\epsilon_{i}^{n}$-consistent price system where $\epsilon_{i}^{n}$ satisfies $\left(1+\lambda_{t}^{i, b}\right)^{-1} \leq 1-\epsilon_{i}^{n}<1+\epsilon_{i}^{n} \leq\left(1+\lambda_{t}^{b, i}\right)$. Precisely, applying Lemma 6.2 we obtain for each $i \geq 0$ a process $Y^{i}$ verifying the inequalities

$$
X_{t}^{i}\left(1-\epsilon_{i}^{n}\right) \leq Y_{t}^{i} \leq\left(1+\epsilon_{i}^{n}\right) X_{t}^{i}, \quad i \geq 0
$$

and satisfying the sde $d Y_{t}^{i}=K_{t}^{i} Y_{t}^{i} d t+\sigma^{i} Y_{t}^{i} d \xi_{t}^{i}, i \in \mathrm{N}, \xi_{t}^{i}:=\gamma^{i} W_{t}^{0}+\bar{\gamma}^{i} W_{t}^{i}$ is a standard brownian motion (for $i=0$, we set $\gamma^{i}=1$ ) and $0 \leq K^{i} \leq C^{i, n}$. We first suitably choose the constants $C^{i}=C^{i, n}>0$ independently of $n$ and small enough for $i \geq 1$. Then, we fix $C^{0, n}$ (depending on $n$ ) sufficiently small so that

$$
\left(\frac{K_{t}^{0}}{\sigma^{0}}\right)^{2}+\sum_{i=1}^{n}\left(\frac{K_{t}^{i}-\beta_{i} K_{t}^{0}}{\sigma^{i} \bar{\gamma}^{i}}\right)^{2} \leq \sum_{i=0}^{n} x_{i}, \quad \forall n
$$

where $\left(x_{i}\right)$ is an arbitrary but fixed summable sequence. Then, the following condition holds:

$$
\int_{0}^{T} \sup _{n}\left[\left(\frac{K_{t}^{0}}{\sigma^{0}}\right)^{2}+\sum_{i=1}^{n}\left(\frac{K_{t}^{i}-\beta_{i} K_{t}^{0}}{\sigma^{i} \bar{\gamma}^{i}}\right)^{2}\right] d t<\infty
$$

where $\beta^{i}:=\gamma^{i} \sigma^{i} / \sigma^{0}$. Applying Proposition 8 of [12] to the corresponding sequence of $\lambda$-consistent price systems, we deduce that Property $(c)$ of Proposition 5.4 holds.

imsart-aap ver. 2011/05/20 file: MafeLepinetteOstafe_02.04.tex date: April 3, 2012 
5.3. Two asset model with infinite horizon. Under the variant approach, we consider the example of [12], i.e. the discrete-time model with only two assets, one of which is taken as a numéraire and its price equals 1 over time. The price dynamics of the strictly positive second asset is given by the following relation

$$
X_{i}=X_{i-1}\left(1+\mu_{i}+\sigma_{i} \epsilon_{i}\right), \quad i \geq 1
$$

where $X_{0}>0,\left(\epsilon_{i}\right)_{i \geq 1}$ is a sequence of independent random variables on a probability space $(\Omega, \mathcal{F}, P)$ and taking values in a finite interval $[-N, N]$ with $E \epsilon_{i}=0, E \epsilon_{i}^{2}=1$. The coefficients here are deterministic and $\sigma_{i} \neq 0$ for all $i$. The support of $\epsilon_{i}$ is $\left[\underline{s}_{i}, \bar{s}_{i}\right]$ where $\underline{s}_{i}<0<\bar{s}_{i}$ and we suppose that $\mu_{i}+\sigma_{i} \bar{s}_{i}>0$ and $\mu_{i}+\sigma_{i} \underline{s}_{i}<0$.

For $n \geq 1$, we consider the stochastic basis $\mathcal{B}^{n}=\left(\Omega, \mathcal{F}^{n}, \mathbb{F}^{n}=\left(\mathcal{F}_{i}^{n}\right)_{i \leq n}, P^{n}\right)$ with the 2-dimensional random process $S^{n}=\left(1, X_{i}\right)_{i \leq n}$ where $\mathcal{F}_{0}^{n}=\mathcal{F}_{0}$ is the trivial $\sigma$-algebra, $\mathcal{F}_{i}^{n}=\mathcal{F}_{i}:=\sigma\left(\epsilon_{1}, \ldots, \epsilon_{i}\right)$, and $P^{n}=P \mid \mathcal{F}_{n}^{n}$. We consider the sequence $\mathcal{M}=\left\{\left(\mathcal{B}^{n}, S^{n}, n\right)\right\}$ of large security markets associated to the deterministic transaction costs coefficients $\left(\lambda_{i}^{0,1}=0, \lambda_{i}^{1,0}\right)_{i \leq n}$ for the exchanges between the bond and the risky assets $X_{i}$. In a bid-ask model, that means that $X_{i}$ is the ask price at time $i$ and $X_{i}\left(1-\lambda_{i}^{1,0}\right)$ is the bidprice. As in [12], we suppose that each model has an equivalent probability measure $Q$ with $b_{i}:=E_{Q} \varepsilon_{i}$ so that there exists also a strictly consistent price system. In particular, we have $\left|b_{i}\right|<N$.

Before presenting our main result, let us observe that we may rewrite the model under an other probability $P^{n}$ so that we may assume that $\mu_{i} \mu_{i+1}<0$ and $\mu_{1}>0$. Indeed, let us choose $\alpha_{i} \in\left(b_{i}, \bar{s}_{i}\right)$ if $i$ is odd and $\alpha_{i} \in\left(\underline{s}_{i}, b_{i}\right)$ otherwise. As $P\left(\epsilon_{i}-\alpha_{i}>0\right)>0$ and $P\left(\epsilon_{i}-\alpha_{i}<0\right)>0$ for all $i$, there exists $P^{n} \sim P$, with $d P^{n}:=\prod_{i=1}^{n} f_{i}\left(\epsilon_{i}-\alpha_{i}\right) d P$ and $E_{P} f_{i}\left(\epsilon_{i}-\alpha_{i}\right)=1$, such that $E_{P} f_{i}\left(\epsilon_{i}-\alpha_{i}\right) \epsilon_{i}=\alpha_{i}$ (see [12]). We then deduce that

$$
\frac{X_{i}}{X_{i-1}}=1+\widetilde{\sigma}_{i} \widetilde{\epsilon}_{i}+\widetilde{\mu}_{i}
$$

where $\widetilde{\sigma}_{i} \widetilde{\epsilon}_{i}:=\sigma_{i} \epsilon_{i}+\mu_{i}-E_{P^{n}}\left(\sigma_{i} \epsilon_{i}+\mu_{i}\right), \widetilde{\mu}_{i}:=E_{P^{n}}\left(\sigma_{i} \epsilon_{i}+\mu_{i}\right)=\sigma_{i} \alpha_{i}+\mu_{i}$ and $E_{P^{n}} \widetilde{\epsilon}_{i}^{2}:=1$. Since $\mu_{i}+\sigma_{i} \bar{s}_{i}>0$ and $\mu_{i}+\sigma_{i} \underline{s}_{i}<0$, we can choose $\left|\alpha_{i}\right|$ large enough such that $\widetilde{\mu}_{i}>0$ if $i$ is odd and $\widetilde{\mu}_{i} \leq 0$ otherwise. Observe that the random variables $\left(\epsilon_{i}\right)_{i \leq n}$ are still independent under $P^{n}$ and so do $\left(\widetilde{\epsilon}_{i}\right)_{i \leq n}$. We denote by $\widetilde{\mu}_{i}, \widetilde{\sigma}_{i}$ and $\widetilde{b}_{i}$ the coefficients of the model when we write it under $P^{n}$. Let

$$
b_{i}:=-\frac{\mu_{i}}{\sigma_{i}}, \quad D_{0, n}^{2}:=\sum_{i=1}^{n} b_{i}^{2}, \quad D_{n}^{2}:=\sum_{i=1}^{n} \widehat{b}_{i}^{2}
$$

imsart-aap ver. 2011/05/20 file: MafeLepinetteOstafe_02.04.tex date: April 3, 2012 
where $\widehat{b}_{i}:=b_{i}-\Delta_{i}$ and $\Delta_{i}:=0$ if $b_{i}=0$, otherwise:

$$
\Delta_{i}:=\mu_{i}^{-1}\left(\Lambda_{i}^{l}-1\right) b_{i}, \text { if } b_{i}>0, \Delta_{i}:=\mu_{i}^{-1}\left(\Lambda_{i}^{r}-1\right) b_{i}, \text { if } b_{i}<0
$$

with $\Lambda_{i}^{r}:=\Lambda_{i}:=\left(1+\lambda_{i}^{10}\right), \Lambda_{i}^{l}:=\Lambda_{i}:=\left(1+\lambda_{i-1}^{10}\right)^{-1}, \lambda_{0}^{10}:=0$.

We also define the analogous coefficients $\left(\widehat{\widetilde{b}}_{i}\right)$ we deduce from $\left(\widetilde{b}_{i}\right)$ and $\left(\widetilde{\mu}_{i}\right)$. Then,

$$
\begin{aligned}
X_{i} & =X_{i-1}\left(1+\sigma_{i}\left(\epsilon_{i}-b_{i}\right)\right), & & i \geq 1, \\
& =X_{i-1}\left(1+\widetilde{\sigma}_{i}\left(\widetilde{\epsilon}_{i}-\widetilde{b}_{i}\right)\right), & & i \geq 1 .
\end{aligned}
$$

At last, we suppose that $b_{i} \widehat{b}_{i} \geq 0$ and so $-\underline{s}_{i}<\widehat{b}_{i}<\bar{s}_{i}$ meaning that the transaction costs coefficients are small enough.

Proposition 5.6.

(a) $D_{\infty}^{2}<\infty \Leftrightarrow N A A 1$;

(b) $D_{\infty}^{2}=\infty \Leftrightarrow S A A 1$.

Proof. It suffices to show the following implications:

(a) If $D_{\infty}^{2}<\infty$, then $\left(P^{n}\right) \triangleleft\left(\overline{\mathcal{Q}}^{n}\right)$ (equivalently $N A A 1$ holds);

(b) If $D_{\infty}^{2}=\infty$, then $\left(P^{n}\right) \triangle\left(\overline{\mathcal{Q}}^{n}\right)$ (equivalently $S A A 1$ holds).

- (a) Notice that in the case where $D_{0, n}^{2}<\infty$, i.e. when the model without friction of [12] does not admit any asymptotic arbitrage opportunity, it is straightforward to conclude using the results of [12] since $\left(X_{i}\right)$ is a strictly consistent price system. The case $D_{0, n}^{2}=\infty$ is the most interesting case; indeed the natural question is how to increase the transaction costs coefficients in order to eliminate an arbitrage opportunity of the frictionless model.

Recall that $\widetilde{\mu}_{1}>0$. For each $n$, we construct a $\lambda$-consistent price system $\left(Y_{i}\right)$ such that $Y_{0}=X_{0}$ and $Y_{i} / Y_{i-1}=\left(X_{i} / X_{i-1}\right) k_{i}$ where $k_{i}>0$ is defined by the relation

$$
k_{i}:=\left(1-\widetilde{\sigma}_{i} \widetilde{\Delta}_{i}\right)^{-1}
$$

i.e. $k_{i}=\left(\Lambda_{i}^{r}\right)^{-1}$ or $k_{i}=\left(\Lambda_{i}^{l}\right)^{-1}$. We have $Y_{i} / Y_{i-1}=1+\widetilde{\sigma}_{i} k_{i}\left(\widetilde{\epsilon}_{i}-\widehat{\widetilde{b}}_{i}\right)$ but also

$$
Y_{i} / Y_{i-1}=1+k_{i} \sigma_{i}\left(\epsilon_{i}-\widehat{b}_{i}\right) .
$$

Recall that $-\underline{s}_{i}<\widehat{b}_{i}<\bar{s}_{i}$. Then, $P\left(\epsilon_{i}-\widehat{b}_{i}>0\right)>0$ and $P\left(\epsilon_{i}-\widehat{b}_{i}<0\right)>0$ for all $i$. It follows that there exists $Q \sim P$ such that $Y$ is a $Q$-martingale. 
Since $\widetilde{b}_{1}<0$, it follows that

$$
\Pi_{j=1}^{i} k_{j}=\left(1+\lambda_{1}^{10}\right)^{-1}\left(1+\lambda_{1}^{10}\right)\left(1+\lambda_{2}^{10}\right)^{-1}\left(1+\lambda_{2}^{10}\right) \cdots
$$

and we get that $\Pi_{j=1}^{i}=1$ or $\Pi_{j=1}^{i}=\left(1+\lambda_{i}^{10}\right)^{-1}$. It follows that

$$
\left(1+\lambda_{i}^{10}\right)^{-1} X_{i} \leq Y_{i} \leq X_{i}
$$

and $\left(Y_{i}\right)$ is a $\lambda$-consistent price system. We then consider the frictionless model of [12] defined by the prices $\left(Y_{i}\right)$ with the coefficients $\left(\widehat{b}_{i}\right)$ in $(5.8)$. Since $D_{\infty}^{2}<\infty$, Proposition 11 (a) of [12] and Proposition 5 of [12] implies the $N A A 1$ condition for our large market defined by $\left(X_{i}\right)$.

- (b) Let us consider an arbitrary sequence of measures $Q^{n} \in \mathcal{Q}^{n}$ associated to the consistent price systems $\left(Z_{i}^{n}\right)_{i<n}$ such that $d Q^{n}=Z_{n}^{0 n} d P^{n}$. Then the real valued process $Y^{n}:=Z^{1 n} / Z^{0 n}$ is a $Q^{n}$-martingale verifying the inequality:

$$
\frac{1}{1+\lambda_{i}^{10}} X_{i} \leq Y_{i}^{n} \leq X_{i}
$$

It follows that $Y_{i}^{n} \leq X_{i} \leq\left(1+\lambda_{i}^{10}\right) Y_{i}^{n}$ and

$$
\frac{1}{\left(1+\lambda_{i-1}^{10}\right)} \frac{Y_{i}^{n}}{Y_{i-1}^{n}} \leq \frac{X_{i}}{X_{i-1}} \leq\left(1+\lambda_{i}^{10}\right) \frac{Y_{i}^{n}}{Y_{i-1}^{n}} .
$$

We deduce that

$$
\frac{1}{\sigma_{i}}\left(\frac{1}{\left.\left(1+\lambda_{i-1}^{10}\right)\right)}-1\right) \leq E_{Q^{n}}\left(\epsilon_{i}-b_{i} \mid \mathcal{F}_{i-1}\right) \leq \frac{\left(1+\lambda_{i}^{10}\right)-1}{\sigma_{i}} .
$$

Consider the case where $b_{i}<0$. Since $\sigma_{i} b_{i}:=-\mu_{i}$ and $\Delta_{i}:=b_{i}-\widehat{b}_{i}$, we get the inequalities

$$
b_{i}\left(\Lambda_{i}^{r}-1-\frac{\mu_{i} \Delta_{i}}{b_{i}}\right) \leq-\mu_{i} E_{Q^{n}}\left(\epsilon_{i}-\widehat{b}_{i} \mid \mathcal{F}_{i-1}\right) \leq b_{i}\left(\Lambda_{i}^{l}-1-\frac{\mu_{i} \Delta_{i}}{b_{i}}\right) .
$$

From $\Delta_{i}=\mu_{i}^{-1}\left(\Lambda_{i}^{r}-1\right) b_{i}$, we deduce that $E_{Q^{n}}\left(\epsilon_{i}-\widehat{b}_{i} \mid \mathcal{F}_{i-1}\right) \leq 0$ and

$$
\widehat{b}_{i} E_{Q^{n}}\left(\epsilon_{i}-\widehat{b}_{i} \mid \mathcal{F}_{i-1}\right) \geq 0
$$

The case $b_{i}>0$ also yields Inequality (5.9). We then deduce that

$$
\operatorname{essinf}_{Q^{n} \in \mathcal{Q}^{n}} E_{Q^{n}}\left(\widehat{b}_{i}\left(\epsilon_{i}-\widehat{b}_{i}\right) \mid \mathcal{F}_{i-1}\right) \geq 0 .
$$

imsart-aap ver. 2011/05/20 file: MafeLepinetteOstafe_02.04.tex date: April 3, 2012 
Let us define the $Q^{n}$-martingale $M^{n}\left(Q^{n}\right)$ by

$$
M_{k}^{n}\left(Q^{n}\right):=\sum_{i=1}^{k}\left[\widehat{b}_{i}\left(\epsilon_{i}-\widehat{b}_{i}\right)-E_{Q^{n}}\left(\widehat{b}_{i}\left(\epsilon_{i}-\widehat{b}_{i}\right) \mid \mathcal{F}_{i-1}\right)\right] .
$$

It satisfies

$$
E_{Q^{n}}\left(M_{n}^{n}\left(Q^{n}\right)\right)^{2}=\sum_{i=1}^{n} E_{Q^{n}}\left[\widehat{b}_{i}\left(\epsilon_{i}-\widehat{b}_{i}\right)-E_{Q^{n}}\left(\widehat{b}_{i}\left(\epsilon_{i}-\widehat{b}_{i}\right) \mid \mathcal{F}_{i-1}\right)\right]^{2} \leq C D_{n}^{2}
$$

where $C$ is a constant. Let us define $M^{n}$ by

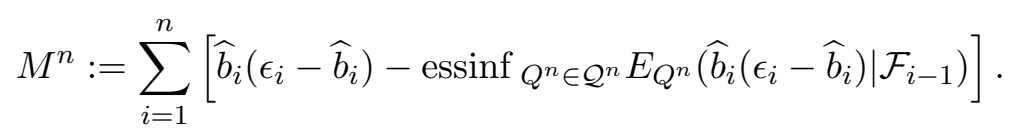

Then, let us introduce the sets $A^{n}:=\left\{-D_{n}^{-3 / 2} M_{n}>1\right\} \in \mathcal{F}^{n}$. Observe that $M^{n} \geq M_{n}^{n}\left(Q^{n}\right)$ for any $Q^{n} \in \mathcal{Q}^{n}$. By the Tchebychev inequality, as $n \rightarrow \infty$, we get that

$Q^{n}\left(A^{n}\right) \leq Q^{n}\left(\left\{-D_{n}^{-3 / 2} M_{n}\left(Q^{n}\right)>1\right\}\right) \leq D_{n}^{-3} E_{Q^{n}}\left(M_{n}^{n}\left(Q^{n}\right)\right)^{2} \leq 4 N^{2} D_{n}^{-1} \rightarrow 0$.

On the other hand, since Inequality (5.10) holds, the complement $\bar{A}^{n}$ of $A^{n}$ verifies

$$
P^{n}\left(\bar{A}^{n}\right) \leq P^{n}\left(\sum_{i=1}^{n} \widehat{b}_{i} \epsilon_{i} \geq\left(D_{n}^{2}-D_{n}^{3 / 2}\right)\right) \leq \frac{4 N^{2} D_{n}^{2}}{\left(D_{n}^{2}-D_{n}^{3 / 2}\right)^{2}} \rightarrow 0 .
$$

Using Proposition 7 [12], we deduce that $\left(P^{n}\right) \triangle\left(\overline{\mathcal{Q}}^{n}\right)$.

REMARK 5.7. Consider a model where $\mu_{i} \mu_{i+1} \leq 0$ for all $i$ and such that $\mu_{i}>0$ and $\mu_{i+1}<0$ implies that $\left(1+\mu_{i+1}\right)\left(1+\mu_{i}\right)=1$, i.e. we have $E_{P}\left(X_{i+1} / X_{i-1}\right)=1$. Assume that $\sum_{i=1}^{\infty} b_{i}^{2}=\infty$, i.e. there is a strong asymptotic arbitrage opportunity in the model without transaction costs. Let us define for $b_{i}>0, \lambda_{i}^{10}=\mu_{i}$. Then the equality $\left(1+\lambda_{i}^{10}\right)^{-1}=1+\mu_{i+1}$ holds and $b_{i+1}<0$, i.e. $\Delta_{i}=b_{i}$ and $\widehat{b}_{i}=0$ for all $i$. We then deduce that there is no more asymptotic arbitrage opportunity.

\section{Appendix.}


6.1. Strictly consistent price systems in the Black-Scholes model.

Let $\left(\Omega, \mathcal{F}, \mathbf{F}=\left(\mathcal{F}_{t}\right)_{t \leq T}, P\right)$ be a stochastic basis where $\left(\mathcal{F}_{t}\right)$ is the natural filtration of a standard brownian motion $W$. Consider the solution $S$ of the sde: $d S_{t} / S_{t}=\sigma d W_{t}+\mu d t$.

Definition 6.1. An $\epsilon$-consistent price system (CPS) is a process $\tilde{S}$ admitting an equivalent martingale measure such that

$$
(1-\epsilon) S_{t} \leq \tilde{S}_{t} \leq(1+\epsilon) S_{t}
$$

By virtue of the martingale representation, a CPS is an Ito process. Consider a strictly consistent price system $\tilde{S}$ (SCPS), i.e. a consistent price system s.t. $(1-\epsilon) S_{t}<\tilde{S}_{t}<(1+\epsilon) S_{t}$. By a measurable selection argument, there is $\alpha_{t} \in L^{0}\left((0,1), \mathcal{F}_{t}\right)$ such that $\tilde{S}_{t}=\alpha_{t}(1-\epsilon) S_{t}+\left(1-\alpha_{t}\right)(1+\epsilon) S_{t}$, i.e.

$$
\tilde{S}_{t}=\left(1+\epsilon-2 \alpha_{t} \epsilon\right) S_{t}
$$

We also have

$$
\alpha_{t}=\frac{1}{2 \epsilon}\left[1+\epsilon-\frac{\widetilde{S}_{t}}{S_{t}}\right]
$$

so that $\alpha$ is also an Ito process. Let us write

$$
d \widetilde{S}_{t} / \widetilde{S}_{t}=H_{t} d W_{t}+K_{t} d t, d \alpha_{t} / \alpha_{t}=\delta_{t} d W_{t}+\gamma_{t} d t
$$

Applying the integration by parts formula, we deduce from (6.1) that:

$$
H_{t}=\sigma-\frac{2 \epsilon \alpha_{t} \delta_{t}}{1+\epsilon-2 \alpha_{t} \epsilon}, K_{t}=\mu-\frac{2 \epsilon \alpha_{t}\left(\delta_{t} \sigma\left(S_{t}\right)+\gamma_{t}\right)}{1+\epsilon-2 \alpha_{t} \epsilon} .
$$

Let us now write $\alpha_{t}:=e^{-X_{t}}$ where $X_{t}>0$ satifies the sde:

$$
d X_{t} / X_{t}=A_{t} d W_{t}+B_{t} d t
$$

We then deduce that

$$
\begin{aligned}
H_{t} & =\sigma-\frac{2 \epsilon \alpha_{t} \ln \left(\alpha_{t}\right) A_{t}}{1+\epsilon-2 \alpha_{t} \epsilon} \\
K_{t} & =\mu-\frac{2 \epsilon \alpha_{t}\left(B_{t} \ln \left(\alpha_{t}\right)+\frac{1}{2} \ln ^{2}\left(\alpha_{t}\right) A_{t}^{2}+A_{t} \ln \left(\alpha_{t}\right) \sigma\left(S_{t}\right)\right)}{1+\epsilon-2 \alpha_{t} \epsilon} .
\end{aligned}
$$

From there, characterizing the set of all SCPS is equivalent to find the set of all processes $(A, B)$ so that there is a change of measure for $\tilde{S}$, defined 
via $H$ and $K$ in terms of $A$ and $B$, under which it is a martingale. Observe that if $\tilde{S}$ is an $\epsilon^{\prime}$-CPS where $\epsilon^{\prime}<\epsilon$, then $\tilde{S}$ is also an $\epsilon$-CPS.

Consider the case $A=0$ and $B_{t}=B=c s t e$. We get that $H_{t}=\sigma$. Since $X_{t}=X_{0} e^{B t}$, we deduce that

$$
K_{t}=\mu+\frac{2 \epsilon X_{0} e^{-X_{0} e^{B t}} B e^{B t}}{1+\epsilon-2 e^{-X_{0} e^{B t}} \epsilon}, \quad t \leq T
$$

Recall that we may replace $\epsilon$ by $\epsilon^{\prime}<\epsilon$ if necessary for finding a SCPS.

With $B<0$, we get that

$$
K_{t}=\mu-\frac{2 \epsilon X_{0} e^{-X_{0} e^{B t}}|B| e^{B t}}{1+\epsilon-2 e^{-X_{0} e^{B t}} \epsilon}, \quad t \leq T .
$$

Lemma 6.2. Assume that the transaction cost coefficient is $\epsilon_{0}>0$. Then, for any $C>0$, there exists $\epsilon \in\left(0, \epsilon_{0}\right)$ and an $\epsilon-C P S$ defined by $A=0$ and $B_{t}=B<0, t \leq T$, such that $H_{t}=\sigma$ and

$$
0<K_{t} \leq C, \quad t \leq T
$$

Proof. It suffices to solve the system: $0<g(t) \leq C, \quad t \leq T$ where

$$
g(t):=\mu-\frac{2 \epsilon X_{0} e^{-X_{0} e^{-D t} D e^{-D t}}}{1+\epsilon-2 e^{-X_{0} e^{-D t}} \epsilon}, \quad D:=|B| .
$$

Let us study

$$
h(y):=\mu-\frac{2 \epsilon X_{0} e^{-X_{0} y} D y}{1+\epsilon-2 e^{-X_{0} y} \epsilon}, \quad y \in\left[e^{-D T}, 1\right] .
$$

The first derivative is

$$
h^{\prime}(y)=-\frac{2 D \epsilon e^{-X_{0} y} f(y)}{\left(1+\epsilon-2 e^{-X_{0} y} \epsilon\right)^{2}}
$$

where $f(y):=1-X_{0} y+\epsilon-\epsilon X_{0} y-2 \epsilon e^{-X_{0} y}$. We get that

$$
f^{\prime}(y)=-X_{0}\left(1+\epsilon-2 \epsilon e^{-X_{0} y}\right)
$$

which is negative under the condition $\epsilon<1$ since $X_{0}>0$. It follows that $f(y) \leq f\left(e^{-D T}\right)$ where

$$
f\left(e^{-D T}\right)=(1+\epsilon)\left(1-X_{0} e^{-D T}\right)-2 \epsilon e^{-X_{0} e^{-D T}} \leq 0
$$

imsart-aap ver. 2011/05/20 file: MafeLepinetteOstafe_02.04.tex date: April 3, 2012 
provided that $X_{0} \geq e^{D T}$ or

$$
\frac{1+\epsilon}{2 \epsilon} \leq \frac{e^{-X_{0} e^{-D T}}}{1-X_{0} e^{-D T}}, \quad X_{0} \leq e^{D T} .
$$

We deduce that $h$ is no-decreasing under the conditions above. It remains to solve the following system:

$$
\begin{aligned}
\frac{2 \epsilon X_{0} e^{-X_{0}} D}{1+\epsilon-2 e^{-X_{0}} \epsilon} & \geq \mu-C, \\
\frac{2 \epsilon X_{0} e^{-X_{0} e^{-D T} D e^{-D T}}}{1+\epsilon-2 e^{-X_{0} e^{-D T} \epsilon}} & <\mu .
\end{aligned}
$$

To solve (6.3), observe that $X_{0} \leq e^{D T}$ implies that

$$
\frac{2 \epsilon X_{0} e^{-X_{0}} D}{1+\epsilon-2 e^{-X_{0} \epsilon}} \geq \frac{2 \epsilon X_{0} e^{-e^{D T}} D}{1+\epsilon}
$$

and the rhs of the inequality above is greater than $\mu-C$ if and only if

$$
X_{0} \geq \frac{(\mu-C)(1+\epsilon)}{2 \epsilon D} e^{D T}
$$

We then set $X_{0}=\left(1-\alpha_{\epsilon}\right) e^{D T}, \alpha_{\epsilon} \in(0,1)$, and we choose $D$ such that the following equality holds:

$$
\frac{(\mu-C)(1+\epsilon)}{2 \epsilon D}=1-\alpha_{\epsilon}
$$

Making $\alpha_{\epsilon}$ converged to 0 , for a given $\epsilon>0$, we get that (6.2) holds since $X_{0} e^{-D T}=1-\alpha_{\epsilon} \rightarrow 1$. Then, (6.4) holds as soon as

$$
2 \epsilon\left(X_{0} e^{-D T}\right) D<\mu(1-\epsilon) .
$$

Since $X_{0} e^{-D T} \rightarrow 1$, it suffices to have $D<\mu(1-\epsilon) /(2 \epsilon)$ where we recall that $D=(\mu-C)(1+\epsilon)\left(1-\alpha_{\epsilon}\right)^{-1} /(2 \epsilon)$. To do so, it is enough that

$$
1-\alpha_{\epsilon}>\frac{(\mu-C)(1+\epsilon)}{\mu(1-\epsilon)}
$$

which is possible, as $\epsilon \rightarrow 0$, since the rhs of the inequality above converges to $(\mu-C) \mu^{-1}<1$. We then conclude. 


\section{References.}

[1] Bouchard B., Chassagneux J.-F. Representation of continuous linear forms on the set of làdlàg processes and the pricing of American claims under transaction costs. Electronic Journal of Probability, 14, 2009, 612-632.

[2] Campi L., Schachermayer W. A Super-replication theorem in Kabanov's model of transaction costs. Finance and Stochastics, 10, 2006, 4, 579-596.

[3] Denis E., Kabanov Y. Consistent price systems and arbitrage opportunities of the second kind in models with transaction costs. Finance and Stochastics., 2010.

[4] De Vallière D., Denis E., Kabanov Yu. Hedging of American options under transaction costs. Finance and Stochastics, 13, 2009, 1, 105-119.

[5] Föllmer H., Schachermayer W. Asymptotic arbitrage and large deviations. Mathematics and Financial Economics, Vol. 1 (3-4), 2008, 213-249.

[6] Guasoni P., Lépinette E., Rásonyi M. The fundamental theorem of asset pricing under transaction costs. Finance and Stochastics., 2012.

[7] Huberman G. A simple approach to arbitrage pricing theory. Journal of Economic Theory, 28 (1), 1982, 183-191.

[8] Klein I., Schachermayer W. Asymptotic arbitrage in non-complete large financial markets. Theory of Probability and Applications, 41 (4), 1996, 927-934.

[9] Klein I. A fundamental theorem of asset pricing for large financial markets. Mathematical Finance 10, 2000,p. 443-458.

[10] Klein I. Free lunch for large financial markets with continuous price processes. The Annals of Applied Probability 13/4, 2003, p.1494-1503.

[11] Kabanov Y.,Kramkov D. Large Financial markets: asymptotic arbitrage and contiguity. Theory of Probability and Applications, 39 (1), 1994, 222-229.

[12] Kabanov Y., Kramkov D. Asymptotic Arbitrage in Large Financial Markets. Finance and Stochastics, Springer-Verlag 1998.

[13] Kabanov Y., Safarian M. Markets with Transaction Costs. Mathematical Theory. Springer, Berlin-Heidelberg-New York, 2009.

[14] Kabanov Y., Stricker C. Hedging of contingent claims under transaction costs. Advances in Finance and Stochastics., Eds. K. Sandmann and Ph. Schönbucher, Springer, 2002, 125-136.

[15] Mbele Bidima Martin L. D., Rásonyi M. On long-term arbitrage opportunities in Markovian models of financial markets. Submitted may 15, 2010, Journal Annals of Operations Research.

[16] Rásonyi M. A remark on the superhedging theorem under transaction costs. Séminaire de Probabilités XXXVII, Lecture Notes in Math., 1832, Springer, BerlinHeidelberg-New York, 2003, 394-398.

[17] Rásonyi M. Arbitrage under transaction costs revisited. Forthcoming in: Optimality and Risk-Modern Trends in Mathematical Finance, ed.: F. Delbaen, M. Rásonyi, Ch. Stricker. Springer, 2009.

[18] Ross, S.A. The arbitrage theory of asset pricing. Journal of Economic Theory, 13 (1), 1976, 341-360.

Ceremade, Place du Maréchal De Lattre De Tassigny, 75775 PARIS CEDEX 16, France

Faculty of Mathematics, University of Vienna, Nordbergstrasse 15, A-1090 Vienna, Austria

E-MAIL: emmanuel.lepinette@ceremade.dauphine.fr; lavinia.ostafe@univie.ac.at

imsart-aap ver. 2011/05/20 file: MafeLepinetteOstafe_02.04.tex date: April 3, 2012 